\author{
Sabine Ladstätter \\ Alfred Galik \\ Daniel Iro \\ Matthias Pfisterer \\ Elisabeth Rathmayr \\ Veronika Scheibelreiter \\ Helmut Schwaiger \\ Michael Teichmann
}

\title{
Die Grabungen des Jahres 2004 im Hanghaus 2 in Ephesos*
}

\section{Einleitung}

Die im Jahr 2004 durchgeführten Grabungen im Hanghaus 2 konzentrierten sich vor allem auf die Wohneinheiten 3 und 5. Nach der Freilegung in den Jahren 1970-72 erfolgten erste punktuelle Untersuchungen unter Bodenniveau im Jahr 1986, als für die Errichtung der ursprünglich vorgesehenen Überdachung des Hanghauses 2 in den beiden Peristylhöfen 16b und 24 Fundamentierungsarbeiten notwendig wurden ${ }^{1}$. Die Grabungen der Jahre 1999-2001 betrafen zum einen Bereiche, in die Fundamente für das neu konzipierte Schutzdach gesetzt werden mussten, zum anderen wurden nach der Entfernung von Mosaikböden die Räume B 17, 16a und 17 flächig freigelegt ${ }^{2}$.

Die 2004 ausgewählten Flächen (Abb. 1) dienten einer Präzisierung des chronologischen Gerüsts sowie einer Dokumentation älterer Bauphasen. Darüber hinaus sollte Fragen zu Funktion und Nutzung einzelner Räume nachgegangen werden. Voraussetzung für die feldarchäologischen Arbeiten war eine professionelle Abnahme der Mosaik- und Ziegelplattenböden in den zu untersuchenden Räumen, wodurch eine flächige Freilegung erst möglich wurde.

Ergänzend zu den Arbeiten in den Wohneinheiten 3 und 5 bedingte die Planung von Besucherstegen eine Grabung im Nordumgang des Peristylhofs 31a der Wohneinheit 6. Hier musste der schlecht erhaltene Marmorboden aus konservatorischen Gründen abgenommen, die gesamte Fläche ausgegraben und in weiterer Folge kompakt verfüllt werden.

\section{Sondage 1/Wohneinheit 3 - Raum 12 ('Musenzimmer’)}

Mit den archäologischen Untersuchungen im Raum 12 der Wohneinheit 3 sollten nähere Aufschlüsse zu den Nutzungsphasen und - soweit möglich - auch zur Funktion des Raumes gewonnen werden. Seit seiner Freilegung im Jahr $1970^{3}$ war im vorgefundenen Ausstattungsensemble der schlichte Bodenbelag aus großfor-

\footnotetext{
* Die Gesamtverantwortung der Grabungen lag bei S. Ladstätter, die Schnittleitungen verteilten sich wie folgt: Sondage 1: D. Iro, V. Scheibelreiter; Sondage 2: E. Rathmayr; Sondage 3: M. Teichmann; Sondage 4a und 4b: D. Iro, H. Schwaiger. Die Münzbearbeitung oblag M. Pfisterer. A. Galik führte eine erste Einordnung der Tierknochen durch. I. Adenstedt, N. Math und N. Pieper danken wir für die graphische Gestaltung der zeichnerischen Dokumentation. K. Herold und P. Pingitzer sei für die konstruktive Zusammenarbeit sehr herzlich gedankt. H. Thür und I. Adenstedt lieferten wichtige Hinweise zum Baubefund. - Die Zitate folgen den Richtlinien der ÖJh 69, 2000, 357 ff. (http://www.oeai.at/publik/autoren.html), die zusätzlich verwendeten Kurzzitate finden sich am Ende des Beitrags.

${ }^{1}$ Durchgeführt von G. Wiplinger. Die daraus geborgenen Funde befinden sich im Etütlük-Depot des Efes Müzesi, Selçuk. H. Vetters, Ephesos. Vorläufiger Grabungsbericht 1986/87, AnzWien 125, 1988, 85-126 bes. 97 f.

2 Die von S. Ladstätter und M. Steskal geleiteten Grabungen sind bislang unpubliziert.

${ }^{3}$ H. Vetters, Ephesos. Vorläufiger Grabungsbericht 1970, AnzWien 108, 1971, 85-101 bes. 94-96.
} 


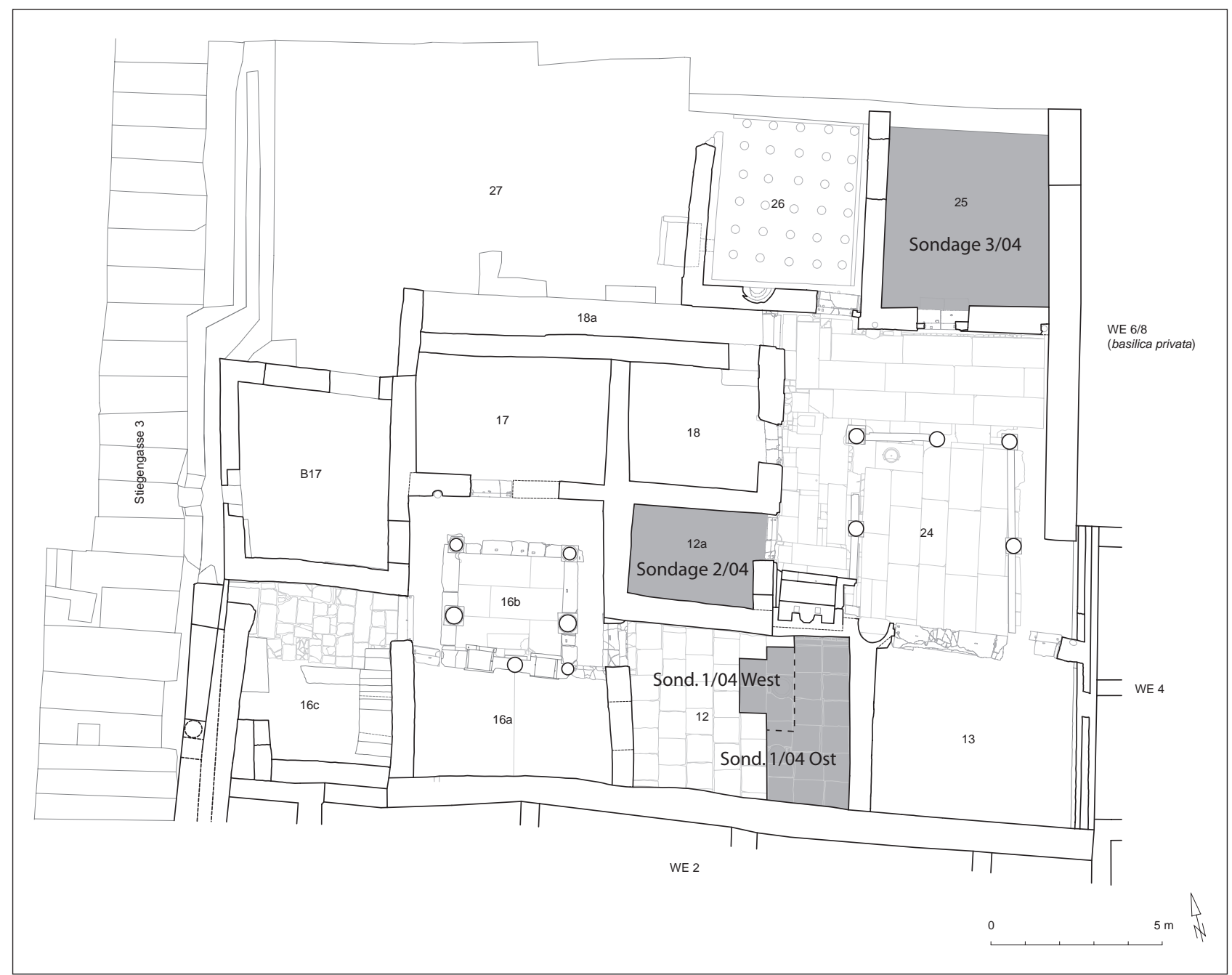

1 Sondagenplan der Grabungen 2004 in den Wohneinheiten 3 und 5

matigen Ziegelplatten mit dem Vorhandensein eines darunter liegenden Hypokaustums begründet worden ${ }^{4}$. Weitestgehend ungeklärt blieb dagegen die Funktion der beiden im östlichen Raumdrittel eingelassenen Säulenbasen ${ }^{5}$ attisch-ionischer Ordnung.

Die Sondage wurde auf der gesamten Raumbreite, ausgehend von der Ostwand bis zur unmittelbar westlich an die Säulenbasen anschließenden Plattenreihe, angelegt. Diese ca. $13 \mathrm{~m}^{2}$ große Fläche weist eine bemerkenswert ungleichmäßige Bodenunterkonstruktion auf. Während der Bodenbelag im westlichen Schnittbereich zwischen SB-S und der Nordwand massiv unterfüllt ist, liegen die Platten im Osten auf einer Abfolge von gemörtelten Ziegelpfeilern auf. Die Grenze verläuft sehr regelmäßig entlang der Ostkanten von SB-S und SB-N (Abb. 2).

\section{Sondage 1/04 Ost}

Das auffallendste Merkmal sind die aufgemauerten Ziegelpfeiler, welche größtenteils gleichartige Konstruktionsweise besitzen: Unter einem Mörtelestrich, der zugleich als Auflager für die Bodenplatten dient, sind jeweils drei Lagen von Ziegeln mit normierten Maßen $(25 \times 12 \times 3 \mathrm{~cm})$ aufgeschichtet. Sie alternieren mit 1-2 cm starken Mörtellagen, deren unterste eine ausgleichende Bettung variabler Höhe ist. Neben den isoliert stehenden, langrechteckigen Pfeilern gibt es zusammengesetzte mit quadratischer Grundfläche. Diese beiden

${ }^{4}$ Strocka, Wandmalerei $126 \mathrm{f}$.

${ }^{5}$ Diese werden im Folgenden als SB-N (nördliche Säulenbasis) und SB-S (südliche Säulenbasis) bezeichnet. 


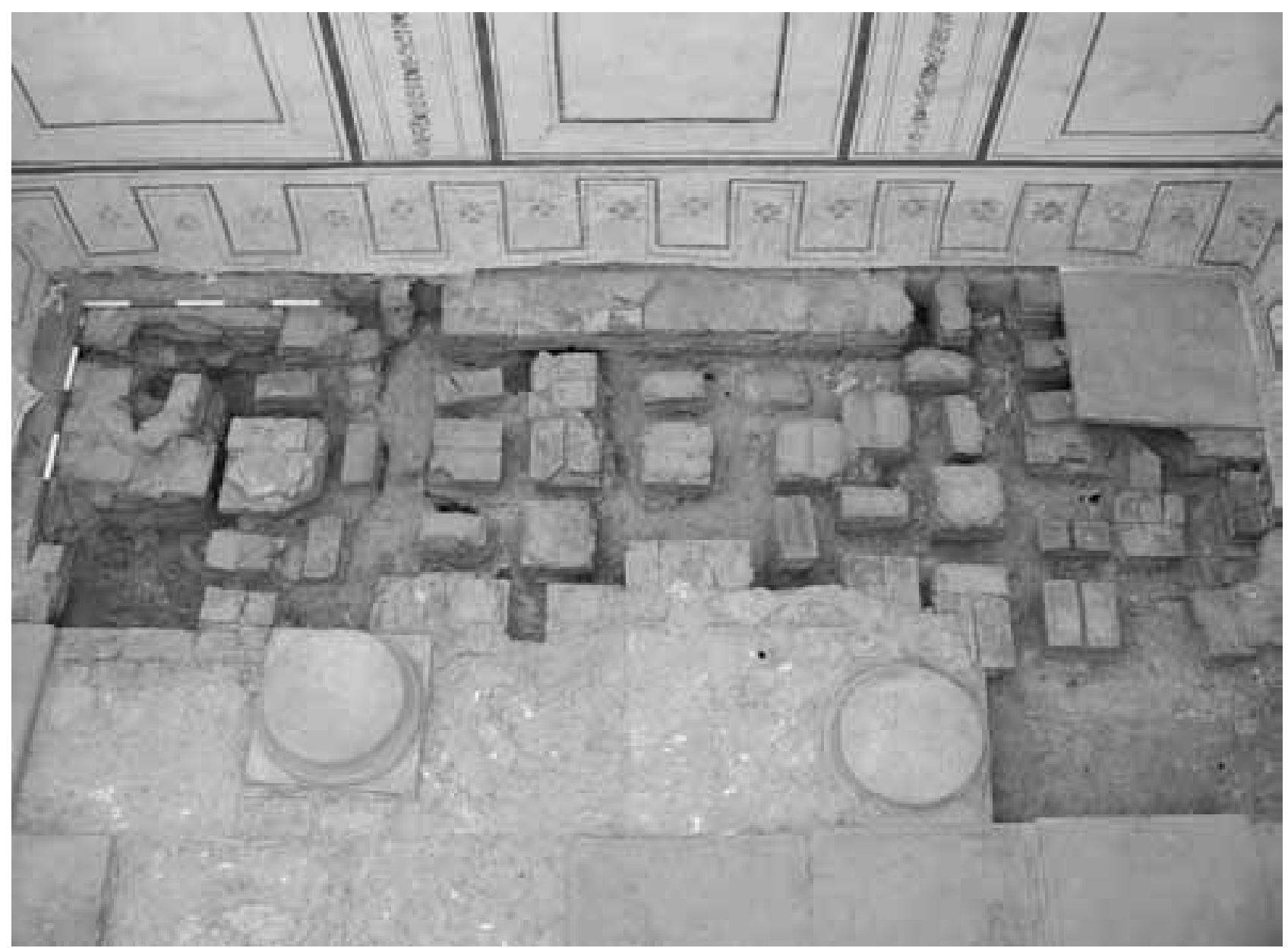

2 Ziegelpfeilersystem in Raum 12 (Sondage 1)

Varianten wechseln ohne ersichtliches System ab, wobei die Pfeiler entsprechend den Raumachsen in je drei ungefähr Ost-West und Nord-Süd orientierten Reihen ausgerichtet sind. In den Zwischenräumen ließen sich Holzkohleablagerungen feststellen, die sich unter den Pfeilern nicht fortsetzten. Sie entstanden demnach nach deren Errichtung, woraus wohl eine ursprüngliche Beheizung des Areals erschlossen werden darf. Vor den Raummauern sind - sieht man von der Südmauer ab - Ziegelsetzungen errichtet, die als Auflager für den Plattenboden dienten. Die größte Struktur ist ein mittig vor die Ostwand gesetztes, 1,7 m langes und $25 \mathrm{~cm}$ breites Ziegelmäuerchen, das aus drei Lagen aufgebaut war und durch eine Schicht aus Kalkmörtel nach oben hin abgestrichen wurde. Als flächiger Untergrund der Ziegelpfeiler ist direkt über dem gewachsenen Fels eine stellenweise nur wenige Zentimeter mächtige Planierung eingebracht (Stratigraphische Einheit [SE] 117). Sie setzt sich aus lehmigem, mit feinkörnigem Felssplitt durchsetzten Material zusammen und war fundleer.

Bei einer im Norden der Sondage aufgedeckten und mit lockerem Sand gefüllten Grube $(\mathrm{Dm} 60 \mathrm{~cm})$ dürfte es sich um eine rezente Störung handeln ${ }^{6}$. Der Bereich wird im Westen von einem dreilagigen Ziegelmäuerchen, das zwischen SB-N und der Nordwand verläuft, abgeschlossen; östlich der Grube lagen wiederum einzelne mit Mörtel gebundene Ziegelpfeiler, die in eine $35 \mathrm{~cm}$ starke, fundleere Aufschüttung (SE 106) gesetzt sind. Hierfür wurden neben den Ziegeln im Standardmaß von $25 \times 12 \times 3 \mathrm{~cm}$ auch solche anderer Dimensionen bzw. Ziegelbruch verwendet. Es ist höchst unwahrscheinlich, dass dieser wenig sorgfältig gestaltete Bereich zur ursprünglichen Hypokaustkonstruktion gehörte, zumal sich die Dichte der Pfeiler nachteilig auf die Luftzirkulation ausgewirkt hätte. Vielmehr ist davon auszugehen, dass es sich um sekundäre Baumaßnahmen handelt, die möglicherweise mit der Verlegung des Plattenbodens in Verbindung standen. Im Süden schließt eine Pfeilerstellung in West-Ost-Richtung an, bei der für den Mittelpfeiler eine mit der Ober-

\footnotetext{
${ }^{6}$ Freundliche Mitteilung K. Herold.
} 


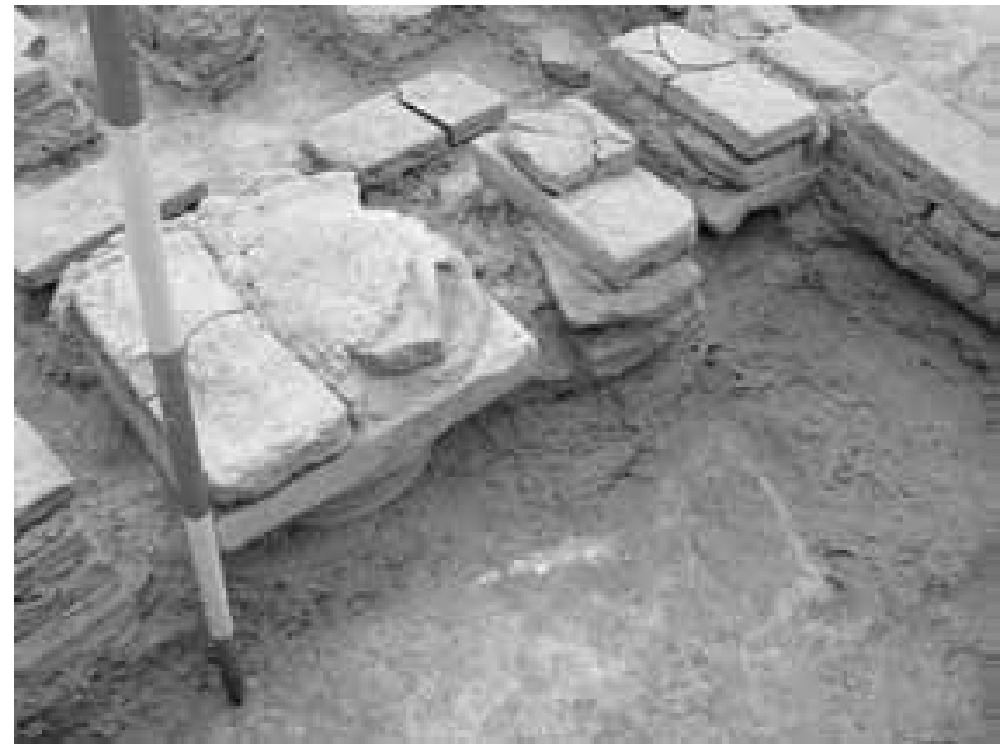

3 Sekundär verwendete attisch-ionische Säulenbasis in Raum 12

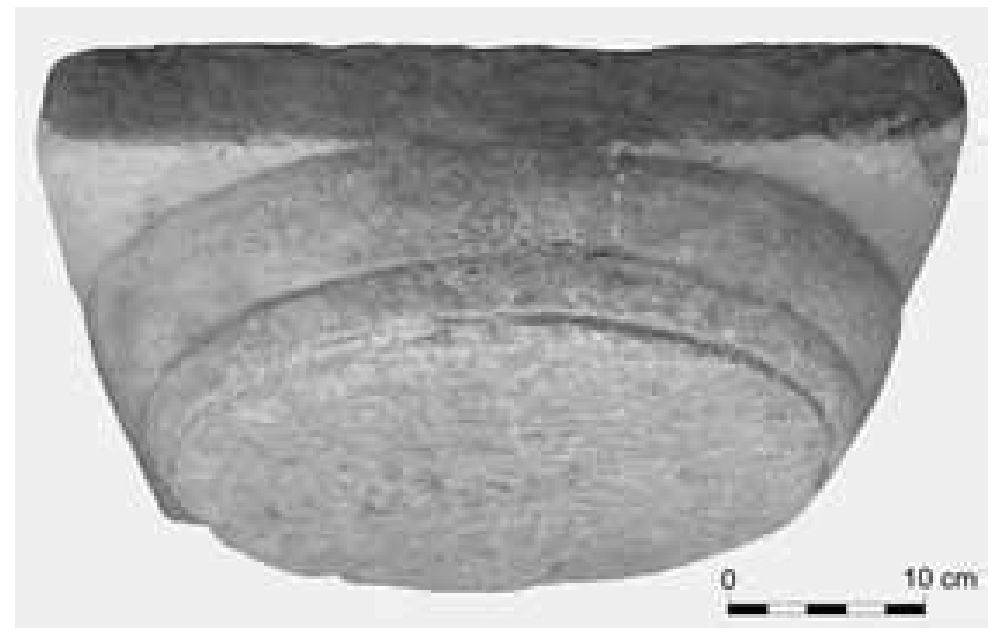

4 Sekundär verwendetes dorisches Kapitell aus Raum 12

seite nach unten vermauerte attisch-ionische Säulenbasis (39,4 × 38,7 × 17,5 cm; oberer Dm $32 \mathrm{~cm}$ ) verwendet wurde (Abb. 3). Auf Spolienverwendung in diesem Bereich deutet ferner die Existenz eines gefalzten Dachziegels. Auch bei den unmittelbar östlich an SB-N und SB-S anschließenden Strukturen dürfte es sich um sekundäre Baumaßnahmen handeln. Dafür spricht einerseits das spoliierte dorische Kapitell (45,7 × 46 × 14,4 cm; unterer Dm 36,8 cm; Abb. 4), andererseits die Dichte der Aufmauerung, die eine Beheizung nur schwer möglich macht. Das Kapitell verdient wegen des seltenen Vorkommens in Ephesos besondere Beachtung; sein profilierter Absatz deutet wahrscheinlich Anuli an, die jedoch nicht fertig ausgeführt worden sind ${ }^{7}$. Aus der Schichtabfolge ist zudem abzulesen, dass die hier aufgemauerten Pfeiler später als die Säulenbasen entstanden sind.

\section{Sondage 1/04 West}

Im Bereich zwischen der Nordwand des Raumes und SB-S lagen die Bodenplatten auf einem dünnen Mörtelestrich (SE 103), der als Ausgleichsschicht für die darunter liegende Rollierung (SE 104) aufgebracht worden war. Diese bestand aus gebrochenen Mauerziegeln - in der Regel weicher gebrannt und größer als die Standardziegel des Hypokaustums -, aus Fragmenten von Tonrohrleitungen sowie marmornen Wandverkleidungsplatten, die in ein Mörtelbett (SE 107) eingetieft worden waren. Im Norden der Sondage wurde unter dieser Rollierung der Fels bei einer Höhe von 29,528 m erreicht. Analog dazu lag auch im Bereich südlich von SB-S der Fels unter einer $5 \mathrm{~cm}$ starken Schicht aus lockerer, fundleerer Erde (SE 114). Dagegen war zwischen den beiden Säulenbasen der Fels abgearbeitet und eine Baugrube ausgehoben worden, in die man die beiden Fundamente gesetzt hatte. Der annähernd quadratische Fundamentsockel der nördlichen Säulenbasis (SB-N; H 65 cm, B 97 cm, T 80 cm) besteht aus vier bis fünf Lagen Bruchsteinen, die mit opus caementitium vergossen wurden (Abb. 5). Beim Eingießen blieben daran Keramikbruchstücke, das Fragment einer profilierten Marmorverkleidungsplatte sowie ein rotgrundiges Wandmalereifragment haften. Auf dem Sockel saß eine durchschnittlich $13 \mathrm{~cm}$ hohe Marmorplatte auf, die als Auflager für die attisch-ionische Säulenbasis $(57,5 \times 58,5 \times 14$ cm; oberer Dm 45,5 cm) diente, mit der sie durch eine $3 \mathrm{~cm}$ starke Mörtelschicht verbunden war.

Eine analoge Fundamentkonstruktion ist auch für SB-S vorauszusetzen, wurde jedoch bei den Grabungen nicht freigelegt. Sichtbar ist lediglich der obere Abschluss durch eine Marmorplatte, auf welche die Basis gesetzt wurde.

\footnotetext{
${ }^{7}$ Außerhalb des Hanghauses 2 kennt man Kapitelle dorischer Ordnung nur von der Neronischen Halle der Tetragonos Agora sowie vom Prytaneion am Staatsmarkt. Freundliche Mitteilung G. Plattner.
} 
H2 WE 3/12

Sondage 1 West

Ost-Ansich

24.06.2004

Aufnahme: $V$. Scheibelreiter

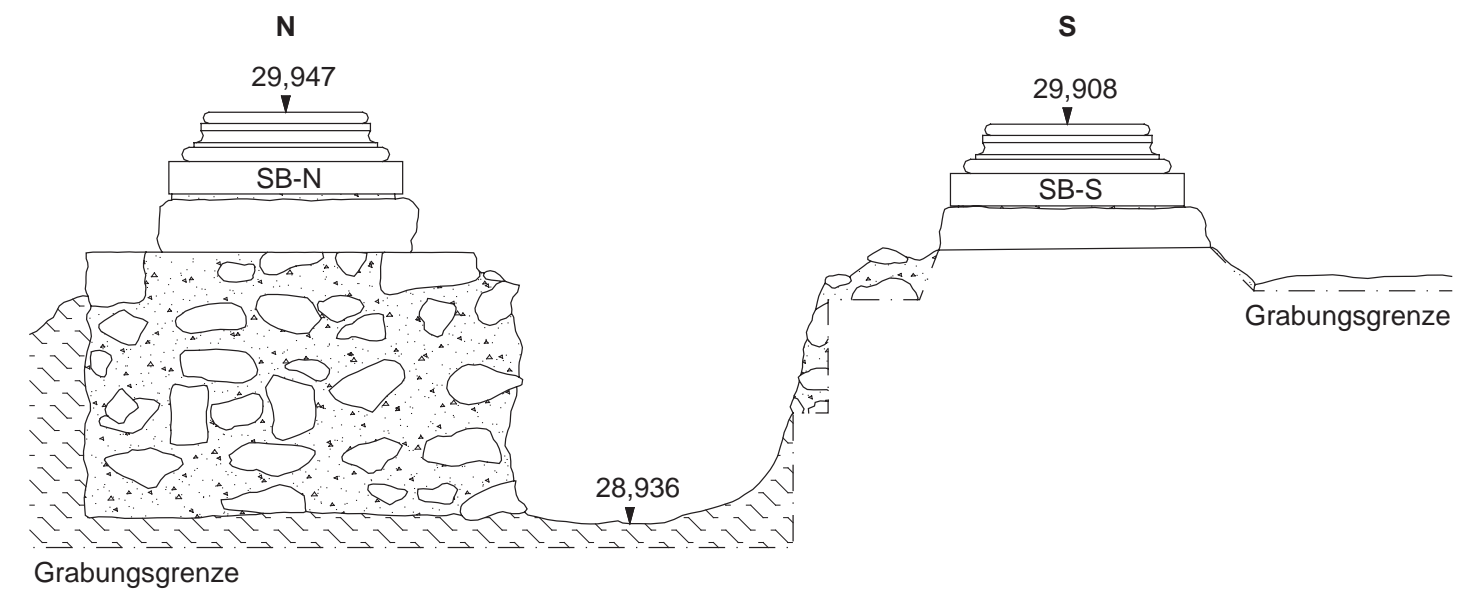

$0,5 \mathrm{~m}$

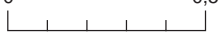

5 Fundamentkonstruktion der Säulenbasen in Raum 12

H2 WE 3/12

Sondage 1 West

Ost-Profil

24.06.2004

Aufnahme: V. Scheibelreiter

Grabungsgrenze

(
Grabungsgrenze

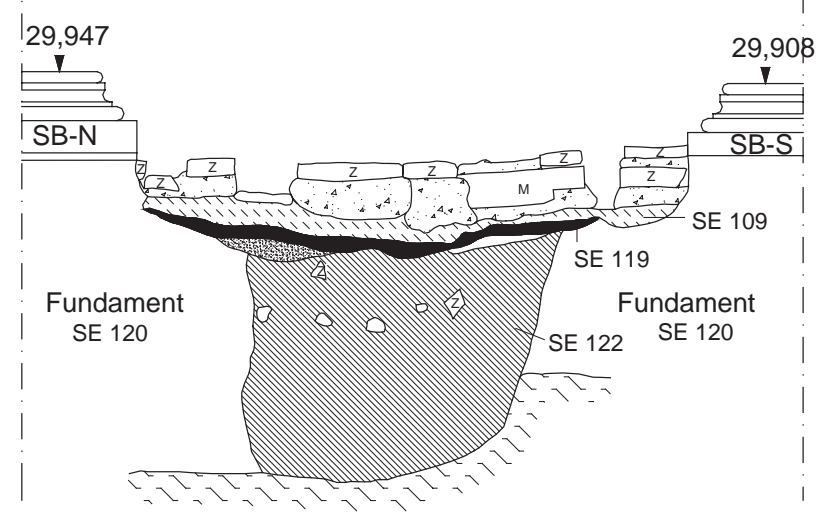

6 Ostprofil über die Baugrubenverfüllung zwischen den Säulenbasen in Raum 12 


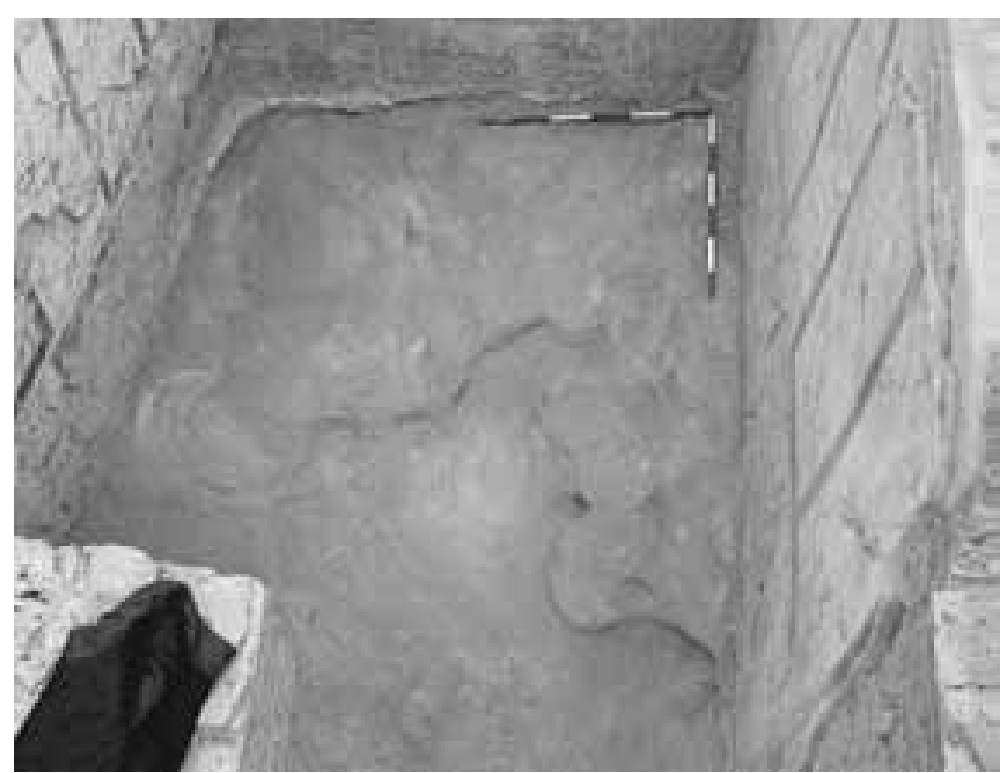

7 Planierung unter dem abgenommenen Mosaikboden in Raum 12a (Sondage 2)
Nach der Fundamentkonstruktion wurde die dafür ausgehobene Baugrube mit Keramik, Erde und Steinen verfüllt. Auch ein im Westen der Fläche liegender, $40 \mathrm{~cm}$ hoher Brekzienblock dürfte im Zuge der Verfüllung eingebracht worden sein (Abb. 6) ${ }^{8}$. Diese bis zu $56 \mathrm{~cm}$ starke Schicht (SE 120) datiert nach einer kursorischen Sichtung des datierenden Fundmaterials in die frühe römische Kaiserzeit. Exemplarisch sei eine EphesosLampe zitiert (Abb. 21, 1; 22, 1), deren Laufzeit vom 1. Jahrhundert v. Chr. bis in augusteische Zeit angenommen werden darf $^{9}$. Eine genaue zeitliche Einordnung des Stratums und damit dessen Zuordnung in Bauphase I oder $\mathrm{II}^{10}$ kann jedoch in jedem Fall erst nach einer genauen Auswertung des Keramikmaterials erfolgen. Auf die Tätigkeit einer Malerwerk-

stätte lassen Ockerklumpen, große Amphorenscherben mit Spuren weißen Kalkfeinputzes an der Innenseite sowie zahlreiche kleinste Muschelbruchstücke schließen. Wie in anderen Bereichen des Hanghauses 2 ist davon auszugehen, dass das nicht mehr benötigte Inventar nach Fertigstellung der Arbeiten an Ort und Stelle entsorgt wurde ${ }^{11}$.

Raum 12 verfügte demnach in einer frühen Bauphase über ein Ziegelpfeilersystem, das aufgrund der hier beobachteten Holzkohle als Hypokaustum in Verwendung stand. Da sich weder ein Präfurnium noch Vorrichtungen für den Luftabzug ${ }^{12}$ an der heute sichtbaren Bausubstanz beobachten ließen, ist das Hypokaustum einer älteren Bauphase zuzuordnen. Der stratigraphische Befund macht deutlich, dass das Hypokaustum spätestens zum Zeitpunkt des Einbaus der Säulenbasen aufgelassen wurde. Abgetragene Pfeiler und einzelne Ziegel, aber auch Architekturspolien, die vermutlich von einer Obergeschossordnung stammen ${ }^{13}$, wurden wieder verwendet und in der Unterkonstruktion des Bodens verbaut.

Die massive Fundamentierung der beiden Säulenbasen schließt wohl aus, dass sie in ihrer originalen Funktion als Träger von Mobiliar oder Statuen dienten ${ }^{14}$. In ihren Dimensionen gleichen sie den Basen aus dem westlich benachbarten Hof 16b, der somit als primärer Aufstellungsort in Betracht zu ziehen ist ${ }^{15}$. In der jüngsten Nutzungsphase des Raumes könnten sie allerdings ihre tragende Funktion verloren und andere Verwendung gefunden haben ${ }^{16}$.

\footnotetext{
${ }^{8}$ Wegen der Sondagengrenzen war nicht festzustellen, ob es sich um einen in situ liegenden Block einer Steinkonstruktion handelt.

${ }^{9}$ Zur Laufzeit von Ephesos-Lampen allgemein s. A. Giuliani, Untersuchungen zu Ephesos-Lampen an Beispielen aus der Werkstatt des Asklepiades, in: F. Krinzinger (Hrsg.), Studien zur hellenistischen Keramik in Ephesos, 2. ErghÖJh (2001) 45-49. Zum Weiterlaufen in augusteischer Zeit s. Ladstätter, WE 4, $235 \mathrm{f}$.

${ }^{10}$ Die Bauphasen im Hanghaus 2 lassen sich folgendermaßen gliedern: Bauphase I - augusteisch-tiberisch; Bauphase I' - Mitte 1. Jh. n. Chr;; Bauphase II - frühes 2. Jh. n. Chr.; Bauphase III - Mitte 2. Jh. n. Chr.; Bauphase IV - spätseverisch; Bauphase IV’ Mitte 3. Jh. n. Chr. s. dazu speziell: Thür, WE 4, 96-101; E. Rathmayr, A.II.2 und B.II.2 Vorbemerkungen zu den Bauphasen, in: Krinzinger, WE 1 und 2; Adenstedt, WE 3 und 5.

11 s. beispielsweise auch die Befunde in Raum 25.

12 Sie waren an den sichtbaren Stellen durchgehend bis etwa auf Höhe der Unterkante der Ziegelpfeiler fein verputzt. Einer Überprüfung entzog sich vor allem der mittlere Abschnitt der Ostwand, dem die Ziegelmauer vorgeblendet war.

13 Ähnliche Proportionen besitzen dorische Kapitelle aus dem Oberstock des Peristyls SR 27 der Wohneinheit 2, die der Bauphase I oder II zuzuordnen sind. Vgl. dazu G. Plattner, B.VII. Architekturdekoration, in: Krinzinger, WE 1 und 2.

${ }^{14}$ Vgl. Strocka, Wandmalerei 126.

${ }^{15}$ Freundliche Mitteilung I. Adenstedt.

${ }^{16}$ Beispielsweise könnten sie zur Aufstellung eines im Raum gefundenen Ziergefäßes aus Marmor gedient haben. Freundliche Mitteilung U. Quatember.
} 


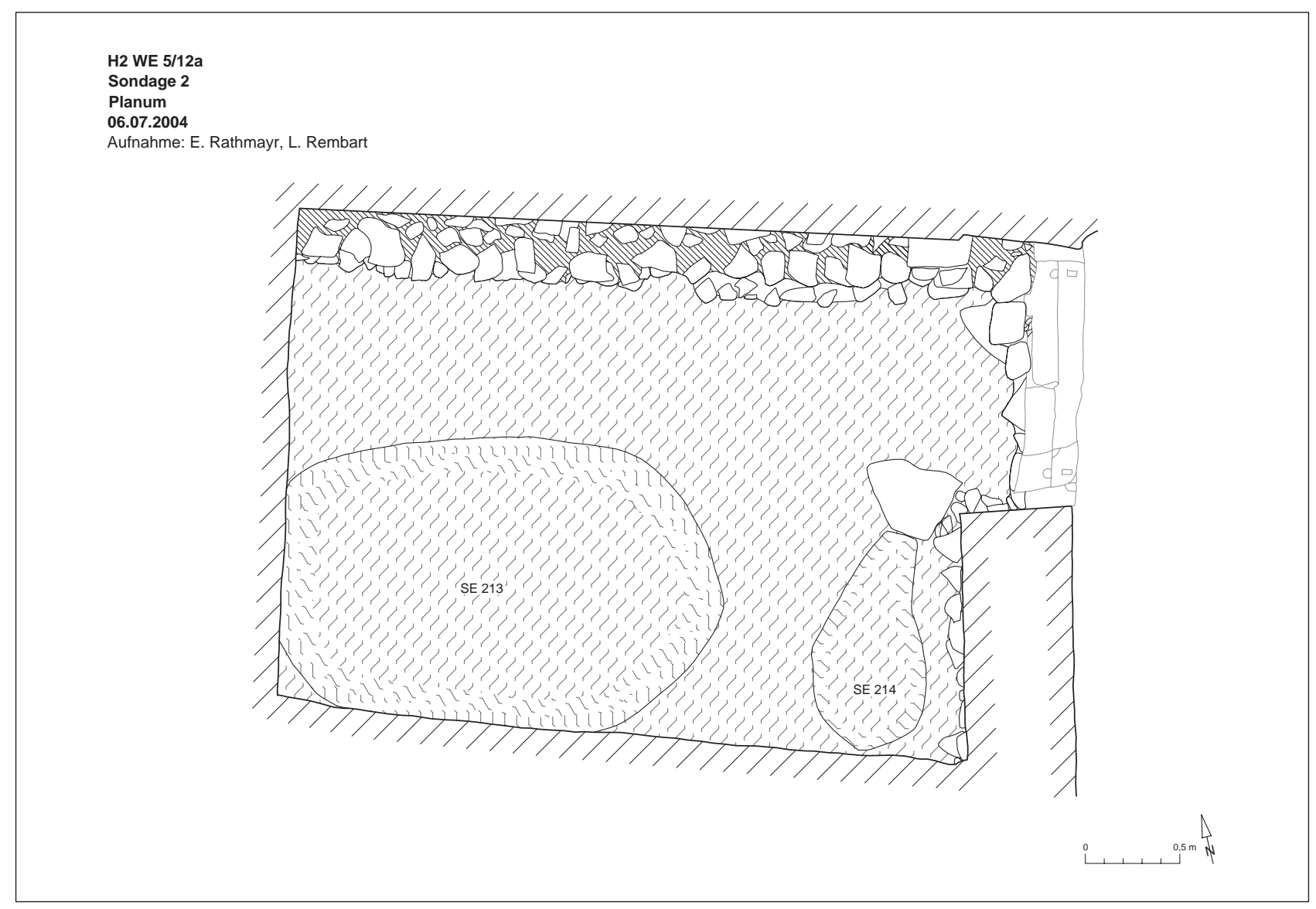

8 Planum in Raum 12a mit hellenistischem Mauerzug und den beiden Gruben

\section{Sondage 2/Wohneinheit 5 - Raum 12a}

Der kleine, als Cubiculum anzusprechende Raum 12a der Wohneinheit 5 war, jedenfalls in der letzten Bauphase, vom Westumgang des Peristylhofs 24 zugänglich ${ }^{17}$. Um Aussagen zu früheren Bauphasen dieses Raumes und der Wohneinheiten 3 und 5 treffen zu können, wurde eine Sondage auf der gesamten Fläche angelegt. Die archäologischen Nachuntersuchungen dienten insbesondere der Frage, ob sich unter dem Mosaikboden Reste eines älteren Peristylhofs, der als Zentrum einer großen, die mittlere Terrasse einnehmenden Wohneinheit anzusprechen wäre, nachweisen lassen ${ }^{18}$. Als ersten Schritt hob man den in diesem Raum verlegten Mosaikboden (SE 201) ab, bei dem es sich um ein grobes, weißes, nur partiell erhaltenes Mosaik handelt. Der Mosaikboden war auf einem nur sehr schlecht erhaltenen, ca. $5 \mathrm{~cm}$ starken Mörtelestrich (SE 202) grauweißer Farbe verlegt. Der Estrich enthielt Keramik, Knochen, Glas, Wandmalereifragmente und Muscheln. Aufgrund des schlechten Erhaltungszustandes der Keramik- und Glasfragmente ist eine zeitliche Einordnung der Mörtelkonstruktion und damit auch der Verlegung des Mosaikbodens nur sehr schwer möglich. Die Funde weisen jedoch allgemein in die frühe bis mittlere Kaiserzeit, ein Zeitansatz, der durch eine Prägung des Kaisers Hadrian (M 31) präzisiert wird. Das Paviment dürfte demnach im Zuge von Umbauarbeiten während Bauphase III entstanden sein.

Unter der Mörtelbettung befand sich eine ca. 12 cm starke Planierung (SE 203) aus lockerer, feiner Erde und kleineren Bruchsteinen, welche die Unterkonstruktion für die Verlegung des Bodens bildete (Abb. 7).

17 Zur Wohneinheit 5 s. Adenstedt, WE 3 und 5.

${ }^{18}$ H. Vetters, Zur Baugeschichte der Hanghäuser, in: W. Jobst, Römische Mosaiken aus Ephesos, FiE VIII 2 (1977) 26 nahm die Teilung einer größeren Wohneinheit in zwei kleinere Wohneinheiten an, wobei »die Wände zwischen den Zimmern 13 und 12 bzw. 12a und 18 zu 17 und 16 die Trennung der Wohnungen herstellten«. 


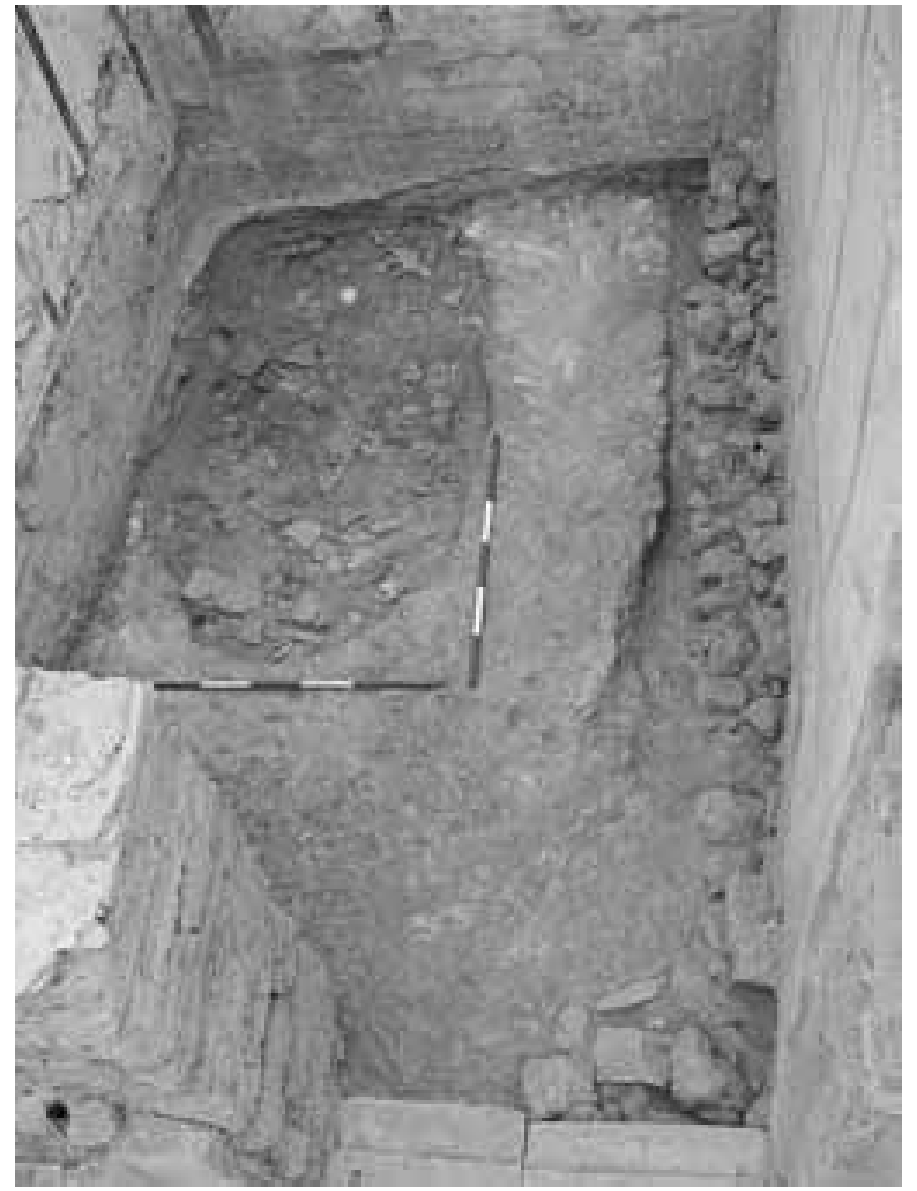

9 Raum 12a, Grubenverfüllung und hellenistischer Mauerzug
Diese Planierung bedeckte einen parallel zur Nordmauer verlaufenden Mauerzug (SE 204), der sich nach Westen fortsetzt und im Nordosten über Eck entlang der Ostmauer des Raumes verläuft (Abb. 8). Dieser wird von der späteren Nordmauer des Raumes partiell überbaut, wodurch nur eine Schale sichtbar ist, während eine zweite vorausgesetzt werden muss. Die OstWest und Nord-Süd orientierten Mauerzüge sind maximal drei Lagen hoch erhalten und bestehen aus kleineren, lehmgebundenen Bruchsteinen $(22 \times 24 \mathrm{~cm}, 15 \times 20 \mathrm{~cm}, 12 \times 13 \mathrm{~cm})$. Das einlagige Fundament dieser Mauer, das entlang der Nordmauer des Raumes ergraben werden konnte, bestand ebenfalls aus kleineren, lehmgebundenen Bruchsteinen. Für die Errichtung wurde eine Baugrube ausgehoben, von der sich ein durchschnittlich $14 \mathrm{~cm}$ breiter Streifen erhalten hat (SE 205). Nach Errichtung der Mauer verfüllte man die Grube mit kleinen Bruchsteinen und Erde (Verfüllung ebenfalls SE 205). Im Nordosten des Raumes konnte eine $1 \times 1 \mathrm{~m}$ große und durchschnittlich $18 \mathrm{~cm}$ starke Fläche festgestellt werden, bei der es sich um eine direkt über dem gewachsenen Fels liegende Erdaufschüttung (SE 212) handelt, die an die älteren Mauerzüge anschließt und als Gehniveau interpretiert werden kann. Das nur partiell erhaltene Fundament erschwert eine genaue zeit-

liche Einordnung des Mauerzuges sowie der zugehörigen Schichten, weist aber zweifelsohne in den späten Hellenismus. Das Randfragment eines Tellers mit gedrechseltem Rand, das in der Lehmbindung der Mauer zutage trat, spricht für eine Einordnung nicht vor dem 1. Jahrhundert v. Chr. ${ }^{19}$; diesen Zeitansatz widerlegen auch die Münzen (M 7. 8) nicht.

Mehrere insgesamt ca. 39 cm starke Planierschichten (SE 206, SE 207, SE 210) lagen unter der Planierung des Mosaikbodens (SE 203); mitverfüllt waren Keramik, Knochen, Muscheln, Wandmalereifragmente, einige Bronze- und Eisennägel sowie kleine Glasfragmente. Die Schichten datieren nach Ausweis des Fundmaterials und der Münze in die frühe Kaiserzeit.

Unter der untersten Planierschicht (SE 207) kam eine 1,30 (Nord-Süd) $\times$ 2,20 (Ost-West) m große und 40-57 cm tiefe, in den anstehenden Fels vertiefte, vollständig verfüllte Grube (SE 213 und SE 208) zum Vorschein (Abb. 9). Die Funktion der Grube ist nicht leicht zu bestimmen, weder war sie mit charakteristischem Material ausgekleidet, noch stand sie mit Bauaktivitäten im Raum selbst in Zusammenhang. Es ist daher wohl eher davon auszugehen, dass sie extra für eine Abfalldeponierung ausgehoben worden war. Die Verfüllung (SE 208) enthielt neben lockerer Erde stellenweise viel Asche, verkohlte Holzstücke, sehr viel Keramik, Terrakotten, Wandmalereifragmente, Ziegelfragmente (darunter auch Dachziegel), Glas, Bronzeund Eisennägel sowie ein Beinnadelfragment. Das Erdmaterial aus dieser Grube wurde zur Gänze gesammelt und geschlämmt ${ }^{20}$. Dabei fiel eine Konzentration hauptsächlich kleiner tierischer Reste wie Geflügel- und Fischknochen auf, wodurch eine Bergung von Sedimentproben nötig wurde. Das aus den Flotat- und Sieb-

${ }^{19}$ Zur Chronologie dieser Form s. S. Ladstätter u. a., Ein hellenistischer Brunnen in SR 9c, in: Lang-Auinger, Hanghaus 1, Funde 22-70 bes. 34 .

${ }^{20}$ Eine detaillierte Bearbeitung und Vorlage der archäozoologischen Befunde soll 2006 durch G. Forstenpointner und A. Galik erfolgen. 


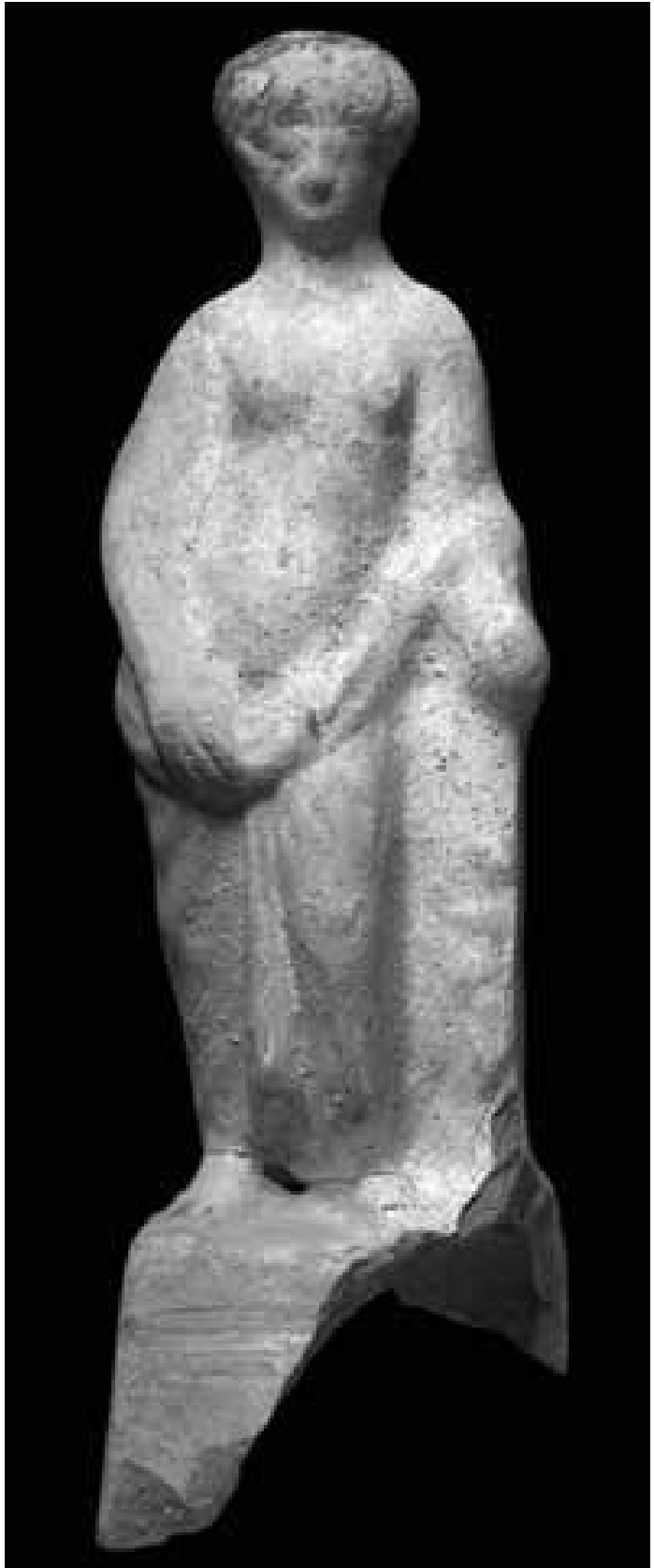

10a Terrakottastatuette aus der Grubenverfüllung in Raum 12a, Vorderseite

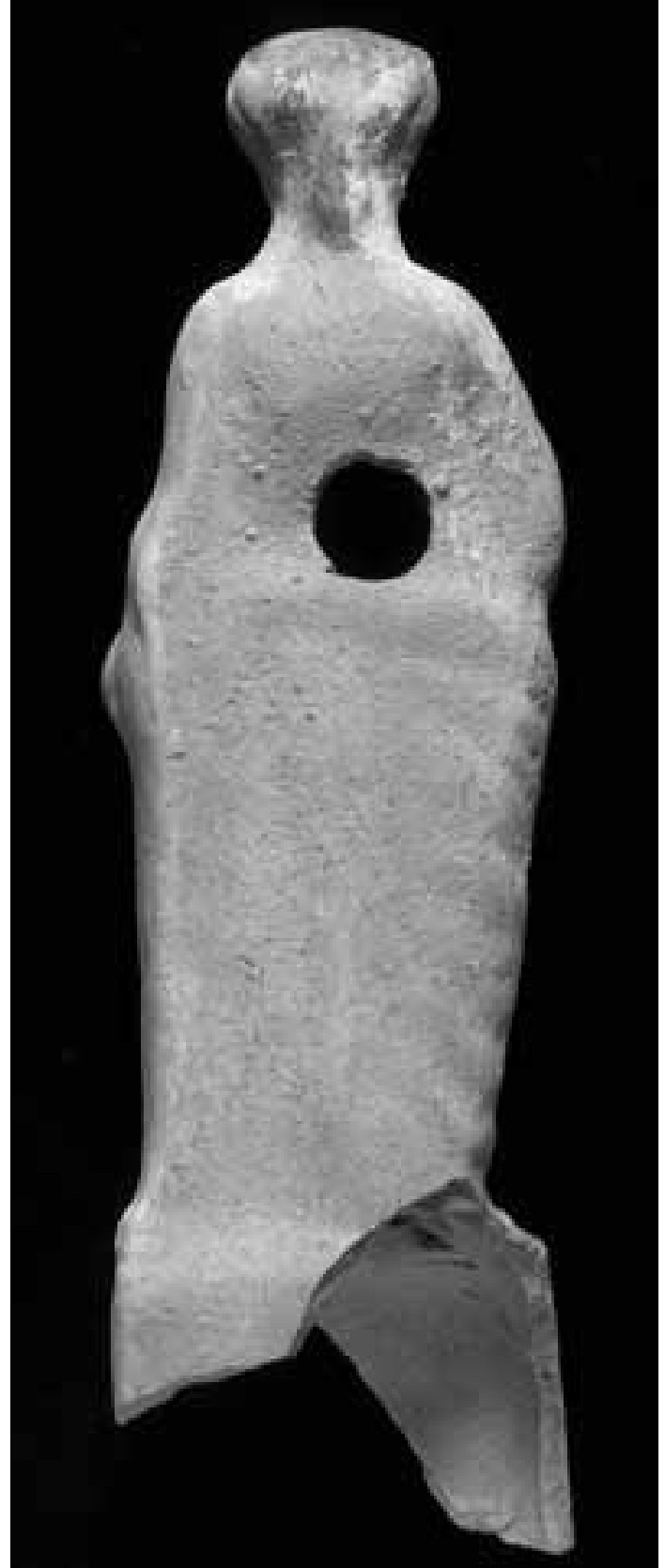

10b Terrakottastatuette aus der Grubenverfüllung in Raum 12a, Rückseite

rückständen gewonnene Material bestätigte die bereits makroskopisch beobachtete, ungewöhnlich hohe Repräsentanz von kleinem tierischem Fundgut. Knochen typischer Nutz- und Haustiere sind unterrepräsentiert und fielen nur in Form klein dimensionierter Reste auf. Daneben fanden sich zahlreiche Knochen von Nagetieren und Amphibien. Eine kursorische Durchsicht der Keramikfunde erbrachte den Nachweis, dass es sich weitgehend um zu Bruch gegangene Ganzgefäße handelt, die hier eingeschüttet wurden. Neben Sigillaten, dünnwandigen und bleiglasierten Gefäßen sowie Küchen- und Gebrauchskeramik ist der hohe Anteil an Amphoren hervorzuheben ${ }^{21}$. Ferner ist auf eine beinahe vollständig erhaltene Terrakottastatuette hinzuweisen (Abb. 10a. b). Die Figur ist aus feinkörnigem Ton mit einem hohen Glimmeranteil hergestellt und weist inklusive Basis eine Höhe von $19 \mathrm{~cm}$ auf. Statuette und Basis sind innen hohl, der Ton ist auf der Innenseite

${ }^{21}$ Eine Bearbeitung des Fundkomplexes soll ebenfalls 2006 erfolgen. 


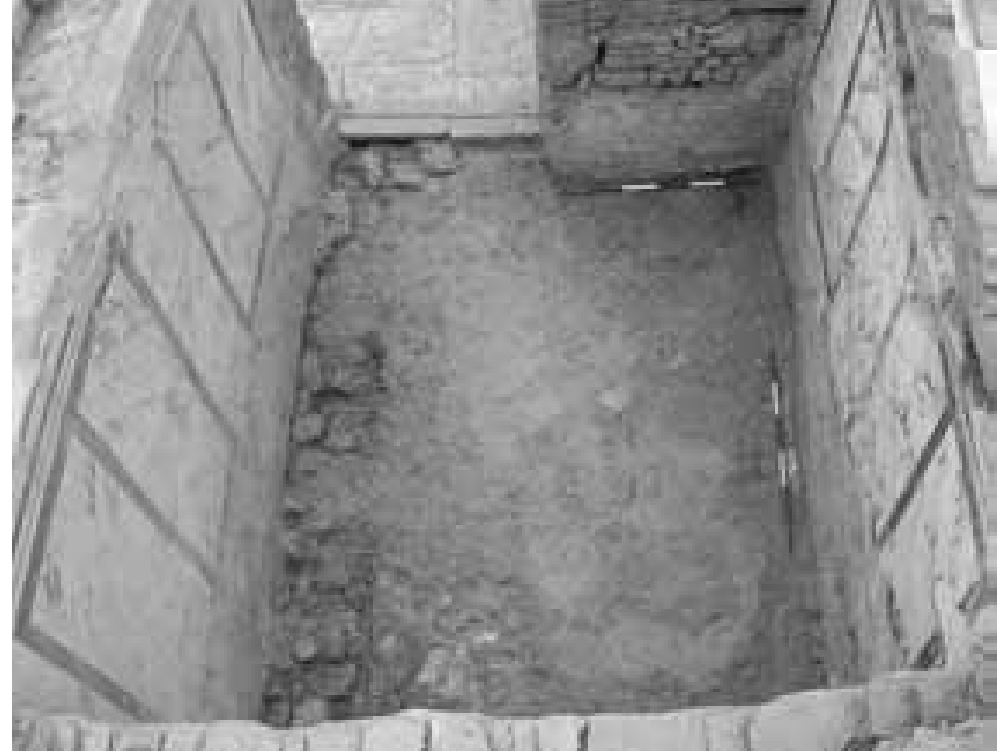

11 Lage des Bauopfers in der Südostecke des Raumes 12a unregelmäßig verstrichen. Auf der Rückseite der Statuette, die abgesehen vom Hinterkopf sehr flach und summarisch ausgeführt ist, befindet sich ein größeres Brennloch (Dm 1,5 cm). Bei der Auffindung waren noch Spuren der einstigen Bemalung erhalten: Körper, Gesicht und Basis waren weiß, Haare schwarz, Brustwarzen und Nabel rosa bemalt. Direkt unterhalb der Brüste verläuft ein breiteres, in rosa Farbe wiedergegebenes Band.

Die weibliche Figur mit linkem Spielund rechtem Standbein steht auf einer hohen Basis. Sie besitzt einen langgestreckten Oberkörper mit hoch sitzenden, kleinen Brüsten. Der Kopf ist nach vorn gerichtet, das lange Haar im Nacken $\mathrm{zu}$ einem Zopf zusammengenommen. Der Unterkörper wird von einem Mantel verhüllt, dessen Enden von der rechten Hand vor der Scham zusammengehalten werden. Neben der linken Körperseite befindet sich ein Pfeiler, auf dem der linke Unterarm aufliegt.

Die Statuette ist in dem reich überlieferten Typus der halbbekleideten Aphrodite, die sich mit dem linken Arm auf einen Pfeiler stützt, dargestellt ${ }^{22}$. Eine Datierung in den späten Hellenismus wird aufgrund der langgestreckten Körperformen mit kleinen, hoch sitzenden Brüsten und ausladenden Hüften vorgeschlagen. Die summarisch ausgeführte Rückseite, an der sich zudem das Brennloch befindet, legt die Aufstellung vor einer Wand oder in einer Nische nahe.

Die zeitliche Einordnung des Fundkomplexes ist unproblematisch, er weist zweifelsohne in augusteischtiberische Zeit. Damit gehört die Aufgabe und Verfüllung der Grube Bauphase I an ${ }^{23}$. Bedeckt wird sie wiederum von der Planierschicht SE 206, womit eine zeitliche Abfolge vorgegeben ist. Möglicherweise handelt es sich um das Inventar eines Haushalts, das nach seiner Zerstörung intentionell eingefüllt wurde. Für einen bewussten Deponierungsvorgang spricht auch die Tatsache, dass sich die Ganzgefäße auf den Grubeninhalt beschränken, wogegen sich in den umliegenden Planierschichten keine anpassenden oder zugehörigen Stücke fanden.

In der Südostecke (Abb. 11) lag, versiegelt durch die Planierung SE 203, ein in die älteste Planierschicht (SE 207) eingetiefter, zur Gänze erhaltener Kochtopf mit Deckel (SE 209, Abb. 12). Es handelt sich um ein Bauopfer, das sorgfältig platziert wurde. Ob der Topf bei seiner Deponierung mit Speisen oder einer Flüssigkeit gefüllt oder leer war, lässt sich nicht mehr feststellen. Die Datierung des grobkeramischen Gefäßes (Abb. 21, 2; 22, 2) ist aufgrund seiner langen Laufzeit nicht unproblematisch. Erstmals treten bauchige Töpfe

${ }^{22}$ LIMC II (1984) Nr. 569-593 mit Abb. s. v. Aphrodite (A. Delivorrias). Unter den Skulpturenfunden aus dem Hanghaus 1 und 2 sind Darstellungen der Göttin Aphrodite sehr häufig, jedoch tritt der Typus der an einen Pfeiler gelehnten hier erstmalig auf; zu den Skulpturenfunden aus dem Hanghaus 1 s. M. Aurenhammer, Skulpturen aus Stein und Bronze sowie C. Lang-Auinger, Terrakotten, in: Lang-Auinger, Hanghaus 1, Funde 153-252 (Skulptur). 209-252 (Terrakotten); zu den Skulpturenfunden aus dem Hanghaus 2 s. E. Rathmayr, Skulpturenfunde der Wohneinheit 4 sowie C. Lang-Auinger, Terrakotten, in: Thür, WE 4, 207-229 (Steinskulptur). 405-408 (Terrakotten); E. Rathmayr, A.XV. Skulpturenfunde der Wohneinheit 1, E. Christof, B.XV. Skulpturenfunde der Wohneinheit 2, C. Lang-Auinger, A.XVI. und B.XVI. Terrakotten, in: Krinzinger, WE 1 und 2. Vgl. dazu die Skulpturenausstattung der Privathäuser von Delos, wo dieser Aphroditetypus nach der sandalenlösenden Aphrodite am häufigsten vorkommt, s. M. Kreeb, Untersuchungen zur figürlichen Ausstattung delischer Privathäuser (1988) 59.

${ }^{23}$ Zur Datierung der Bauphase I im Hanghaus 2 s. Ladstätter, WE 4, 232-237 und S. Ladstätter, A.IX. und B.IX. Archäologische Nachuntersuchungen, in: Krinzinger, WE 1 und 2. Zu einem möglicherweise analogen Befund im Hanghaus 1 s. U. Outschar, Aspekte zur Chronologie, in: C. Lang-Auinger, Hanghaus 1 in Ephesos. Der Baubefund, FiE VIII 3 (1996) 26. 30-31, Fundkomplexe $\mathrm{B} / \mathrm{I}$ und $\mathrm{B} / \mathrm{II}$. 
mit schräg ausladendem Rand und fehlender Standfläche im zweiten Viertel des 1. Jahrhunderts $\mathrm{n}$. Chr. auf ${ }^{24}$, die funktionale Formgebung blieb allerdings bis in das 3 . Jahrhundert n. Chr. unverändert. Auffallend ist auch, dass der Topf der Gattung der ephesischen Küchenkeramik angehört, während der Deckel der Gebrauchskeramik zugeordnet werden kann. Diese Kombination findet sich auch in einem in severische Zeit zu datierenden küchenkeramischen Ensemble der Wohneinheit $1^{25}$. Trotzdem kann das Bauopfer aufgrund seiner stratigraphischen Position in die mittlere römische Kaiserzeit datiert werden, möglicherweise ist es zeitgleich mit der Planierung SE 203 und der Verlegung des Mosaikbodens anzusetzen.

Unter dem Bauopfer (SE 209) bzw. der un-

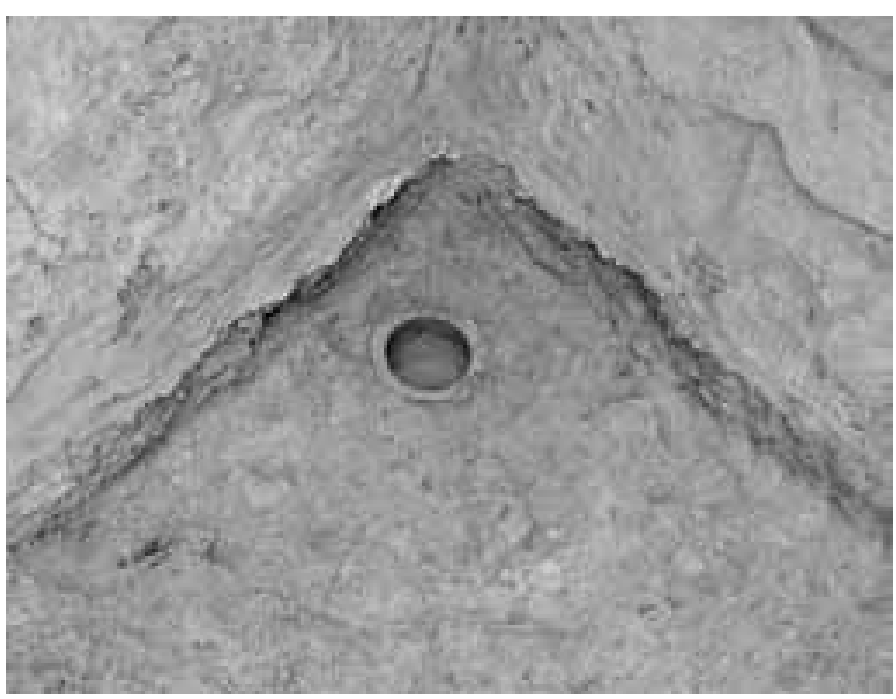

12 Detail des Bauopfers tersten Planierschicht (SE 207) wurde die Erde bis auf den anstehenden Fels abgetragen, wobei im Bereich vor der Ostmauer eine weitere, jedoch kleinere, 1,10 (Nord-Süd) $\times$ 0,80 (Ost-West) $\mathrm{m}$ große und durchschnittlich $27 \mathrm{~cm}$ tiefe, in den anstehenden Fels vertiefte Grube (SE 214) festgestellt wurde (Abb. 8). Ihre Verfüllung (SE 211) ist wenig reichhaltig und auch chronologisch schwer auszuwerten; ebenso wie bei der größeren Grube (SE 213) im südlichen Raumteil muss auch in diesem Fall die Funktion des Grubenaushubs unklar bleiben.

Anschließend wurde der Raum im gesamten Bereich bis auf den anstehenden Fels (SE 215), der aus Serizit-Phyllit (Glimmerschiefer) besteht, freigelegt (Abb. 13). Das nichtabgearbeitete Felsniveau liegt zwischen 29,131 m und 28,707 m.

Als vorläufiges Ergebnis der Sondage im Raum 12a ist festzuhalten, dass die beiden in den anstehenden Fels vertieften Gruben (SE 213 und 214) sowie die freigelegten Nord-Süd und Ost-West orientierten Mauerzüge und das zugehörige Gehniveau (SE 204 und 212) älter sein müssen als die Konstruktion des Raumes selbst. Auf eine hellenistische und damit vorhanghauszeitliche Zeitstellung dieser baulichen Strukturen weisen neben der Mauerwerktechnik (lehmgebundene Bruchsteine) und der Keramik auch die Münzfunde: Eine Münze (M 7) aus der Lehmbindung der Mauer datiert hellenistisch, eine weitere (M 8) aus der Baugrube in das 4./3. Jahrhundert v. Chr. ${ }^{26}$. Bei der Errichtung der Wohneinheit dienten diese älteren Mauerzüge ebenso wie in anderen Wohneinheiten des Hanghauses 2 als Fundamentierung des Mauerwerks der Bauphase I ${ }^{27}$. Der in Ost-West-Richtung ziehende Mauerzug setzt sich nach Westen fort und könnte mit hellenistischen Mauerzügen, die bei früheren Sondagen im Peristylhof der Wohneinheit 3 freigelegt wurden, in Zusammenhang stehen $^{28}$. Auch die in den anstehenden Fels vertieften Gruben (SE 213, SE 214), deren Anlage spätestens in Bauphase I datiert, könnten zeitgleich mit den ältesten baulichen Strukturen entstanden sein. Da eine in der Grubenverfüllung gefundene Münze (M 17) augusteisch datiert und die jüngsten Keramikfragmente der augusteisch-tiberischen Zeit angehören, steht die Verfüllung der Grube in Zusammenhang mit der Anlage des Raumes 12a in Bauphase I. Der bis zur Zerstörung der Wohneinheit im dritten Viertel des 3. Jahrhunderts $\mathrm{n}$. Chr. in Funktion stehende Mosaikboden ist dagegen frühestens der Bauphase III zuzuweisen. Erstaunlich hoch ist der Anteil an hellenistischen Altstücken unter den Münzen in den Planierschichten, datiert doch die jüngste Prägung in die Regierungszeit des Kaisers Hadrian. Diesem Befund widersprechen auch die Keramik- und Glasfragmente nicht, die jedoch aufgrund ihres schlechten Erhaltungszustandes vorläufig nur grob in die

\footnotetext{
${ }^{24}$ Ladstätter, WE 4, K 454.

${ }^{25}$ S. Ladstätter, A.X. Keramik, in: Krinzinger, WE 1 und 2.

${ }^{26}$ s. dazu den Katalog der Münzen am Ende des Beitrags.

${ }^{27}$ Vgl. z. B. die Wohneinheiten 1 und 2 des Hanghauses 2, wo man viele Mauern der Bauphase I auf ältere hellenistische Mauerzüge setzte, s. S. Ladstätter, A.II.1 und B.II.1 Hellenistische Bebauung sowie E. Rathmayr, A.II.2.1 und B.II.2.1 Bauphase I, in: Krinzinger, WE 1 und 2.

${ }^{28}$ Dies kann leider nicht mehr verifiziert werden, da von diesen Grabungen keine Dokumentation vorliegt.
} 


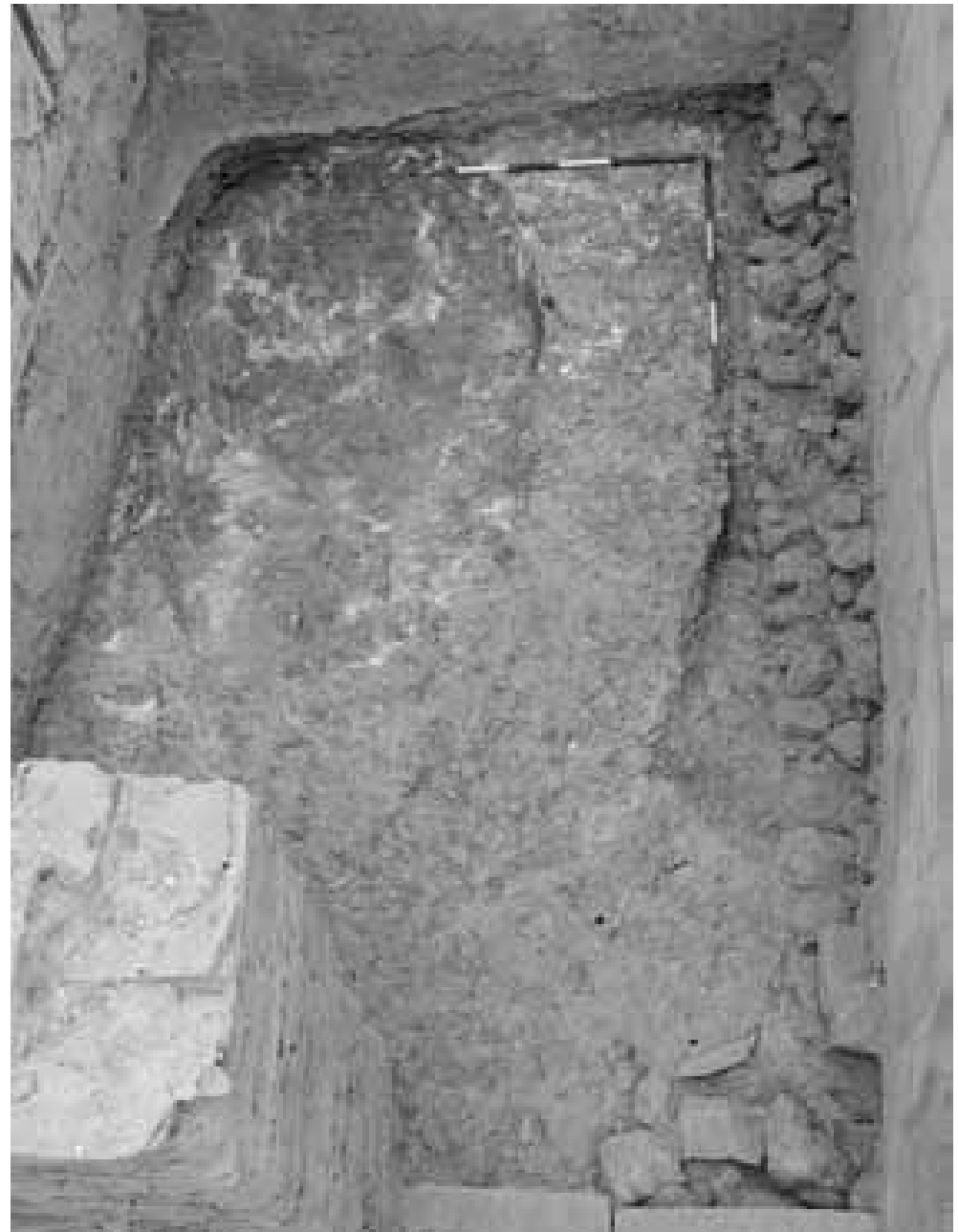

13 Hellenistischer Mauerzug und Grube in der Südwestecke des Raumes 12a frühe bis mittlere Kaiserzeit datiert werden können ${ }^{29}$.

Der küchenkeramische Topf, den man im Zuge der Planierung und Unterkonstruktion des Bodens deponierte, ist wie vergleichbare Beispiele aus den Wohneinheiten 3 und 4 im Hanghaus 2 als Bauopfer zu deuten ${ }^{30}$. Abgesehen von diesen Parallelen spricht für die Interpretation, dass nur im Bereich des Topfes im Mauerprofil eine Kohleschicht und direkt darüber eine Ziegelsplittschicht (je ca. $1 \mathrm{~cm}$ stark) festgestellt wurden. Erste dürfte auf eine kultische Handlung - wahrscheinlich ein Brandopfer - zurückzuführen sein, die Ziegelsplittschicht diente dazu, das Bauopfer zu versiegeln.

Bezieht man die Grabungsbefunde auf die einleitend formulierten Fragestellungen, so konnte eindeutig geklärt werden, dass sich im Bereich von Raum 12a keinerlei Hinweise auf einen älteren Peristylhof finden. Ferner ist ausgeschlossen, dass das 'Musenzimmer' (Raum 12) von Raum 12a aus beheizt worden war. Die hellenistischen Befunde gehören einem Zeitraum an, in dem auf dem Areal des Hanghauses 2 zwar Bauaktivität zu beobachten, diese jedoch in keinen Zusammenhang mit den späteren römischen Wohnbauten zu setzen ist. Die Anlage des

Raumes 12a erfolgte wohl bereits in der frühen römischen Kaiserzeit, das heute sichtbare Paviment erhielt er jedoch erst im Verlauf des 2. Jahrhunderts. In weiterer Folge blieb er bis zu seiner Zerstörung und Aufgabe im dritten Viertel des 3. Jahrhunderts n. Chr. nahezu unverändert.

\section{Sondage 3/Wohneinheit 5 - Raum 25}

Raum 25 der Wohneinheit 5 ist aufgrund seiner Größe $(5,00 \times$ 4,70 m) und Ausstattung, die in der jüngsten Ausstattungsphase aus Marmor imitierender Wandmalerei ${ }^{31}$ und einem polychromen Mosaikboden ${ }^{32}$ bestand, wohl als Repräsentationsraum ${ }^{33}$ anzusprechen. Der Raum war in seiner letzten Bauphase durch eine $90 \mathrm{~cm}$ breite Tür zu betreten. Eine zweistufige, abwärts führende Schwelle glich den Niveauunterschied zum höher liegenden Nordumgang des Peristylhofs 24 aus. Um Aussagen zu früheren Bauphasen dieses Raumes und zur Datierung der östlich an den Raum anschließenden Basilika der Wohneinheit 6 treffen zu können, wurde die

\footnotetext{
${ }^{29}$ Eine präzise Auswertung dieser Funde wird im Sommer 2006 erfolgen.

${ }^{30}$ Zum Bauopfer der Wohneinheit 3, vor der Schwelle von Raum 17 zum Hof 16 s. S. Ladstätter, Zur Datierung des Löwenmosaiks im Hanghaus 2 von Ephesos, in: B. Brandt - V. Gassner - S. Ladstätter (Hrsg.), Synergia. Festschrift F. Krinzinger I (2005) 179-186; zu jenem aus Hof 21 der WE 4 s. Ladstätter, WE 4, 238.

31 Strocka, Wandmalerei 118 Abb. 270-274.

32 Jobst (Anm. 18) 108-109 Abb. 206-207.

${ }^{33}$ Zur Wohneinheit 5 s. Adenstedt, WE 3 und 5.
} 
gesamte Fläche des Raumes ausgegraben. Nach der Abnahme des Mosaikbodens (SE 301) konnten neben kleineren rezenten Störungen (SE 302-306) im West-, Südwest- und Südostbereich (SE 307-309) des Raumes Reste eines Mörtelestrichs nachgewiesen werden. Verschiedene Oberflächenfarben und die Zusammensetzung weisen auf einen unterschiedlichen Erhaltungsgrad eines mehrlagigen, ineinander übergehenden Unterbodens: SE 308 war ein rötlich heller Boden mit viel kleinteiligem Ziegelsplitt und zahlreichen hellen Wandverputzfragmenten, die sich besonders im direkten Vorfeld der Wand häuften. SE 307 hatte eine gräuliche, SE 309 eine dunkle Oberflächenfarbe. Der rote Mörtel ist als rudus, der weiße Mörtel als nucleus des Mosaikbettes anzusprechen (Abb. 14). Diese hier beobachtete Mehrschichtigkeit der Unterkonstruktion des Mosaikbodens entspricht dem auch sonst im Hanghaus 2 gängigen Usus ${ }^{34}$.

Der Fund eines dünnwandigen Bechers (Abb. 21, 3; 22, 3) in SE 309, der Reste roter Farbe enthielt, belegt, dass während der Anlage des Bodens auch die Wände des Raumes neu gestaltet wurden. Die keramischen Funde sowie die jüngste Fundmünze datieren diese Baumaßnahmen in das 3. Jahrhundert und weisen die jüngste Ausstattungsphase und damit auch die rotgrundige Malerei des Raumes Bauphase IV $\mathrm{zu}^{35}$.

Entlang der West- und Ostmauer kam auf der gesamten Länge, direkt an die

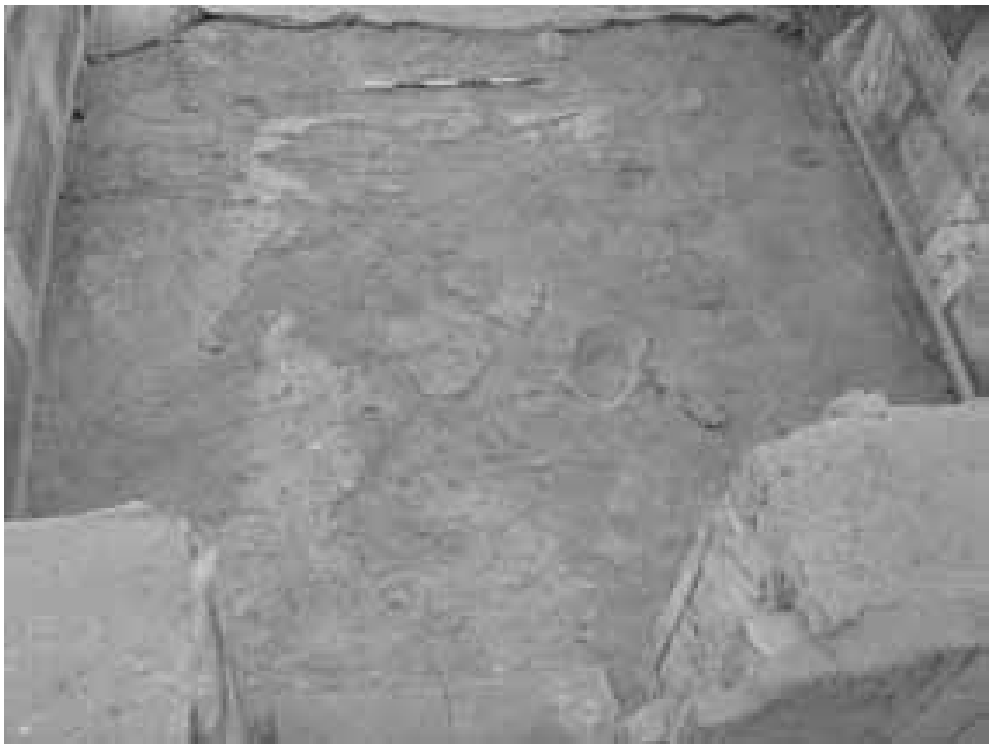

14 Unterkonstruktion des Mosaikbodens in Raum 25 (Sondage 3)

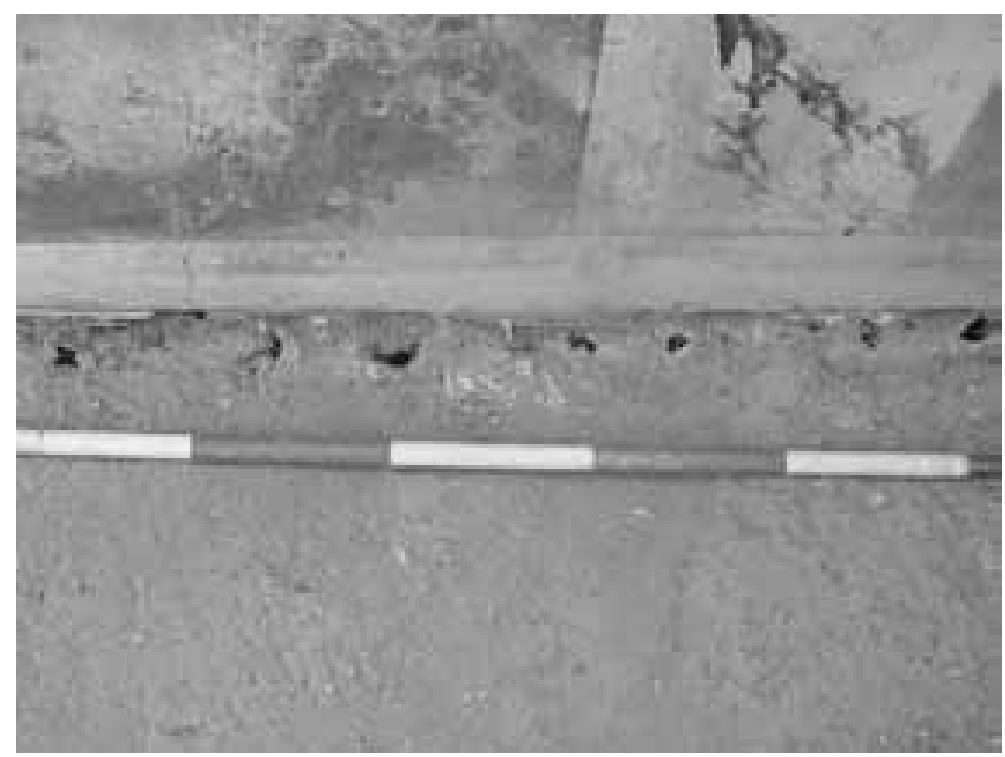

15 Holzkohlestücke entlang der Ostmauer des Raumes 25 Unterkante des Mosaiks anschließend, eine Schicht rötlichen Mörtels mit zahlreichen großen und beinahe regelmäßig gelegten Holzkohleeinschlüssen zutage (SE 310, Abb. 15); sichtbar waren sie lediglich an den Mauerkanten, während sie sich im Rauminneren nicht fortsetzten. Das möglicherweise unter Luftabschluss inkohlte Holz scheint als konstruktives Element unbekannter Funktion verwendet worden zu sein.

Unter den Mörtelschichten SE 307-309 befanden sich zwei Lehmpackungen aus hartem, kompaktem Material: im Ostbereich (SE 311) bestehend aus Lehm mit rötlichen Oberflächenpartien und einem leichten Ost-West-Gefälle, im restlichen Teil des Raumes aus hellem, z. T. leicht rötlichem Lehm (SE 313). Die Kompaktheit lässt darauf schließen, dass es sich bei den Lehmpackungen um ein Arbeitsniveau handelte, zudem bildeten sie eine hervorragende Unterlage für die darüber aufgebrachte Mörtelkonstruktion. Für ein Arbeitsniveau spricht auch eine wenige Millimeter starke Schotterlinse in einem ca. $40 \mathrm{~cm}$ breiten Streifen im Nordbereich des Raumes (SE 312), die direkt auf SE 313 auflag. Das Material diente mit großer Wahrscheinlich-

${ }^{34}$ V. Scheibelreiter, B.VI.5 und VI.6. Mosaikböden, in: Krinzinger, WE 1 und 2.

${ }^{35}$ Eine genaue Bearbeitung des Fundmaterials steht noch aus. 


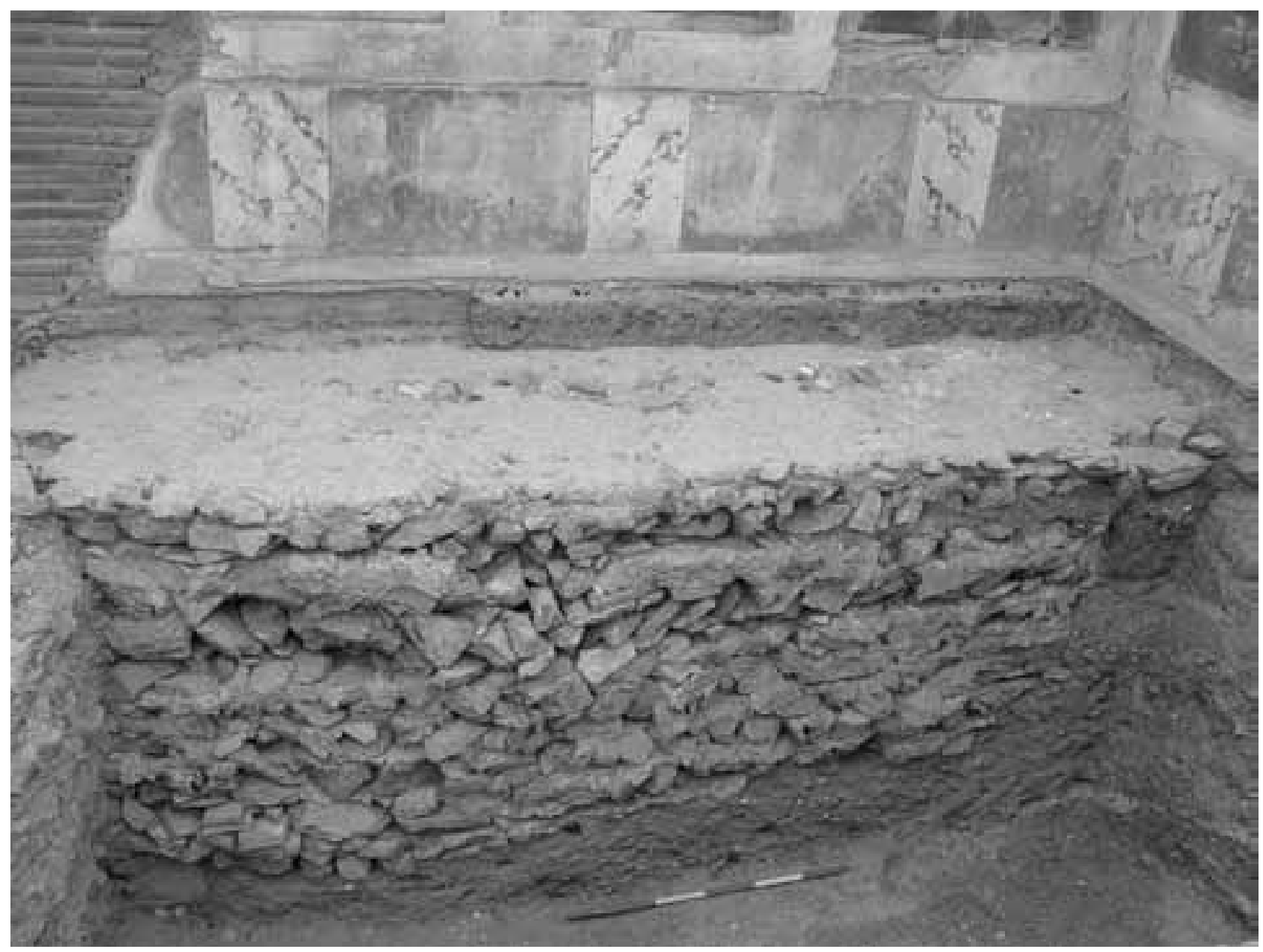

16 Massive Ziegelbruchstein-Packung entlang der Ostmauer des Raumes 25

keit als Zuschlagsstoff bei der Mörtelaufbereitung und wurde nach Abschluss der Arbeiten an Ort und Stelle belassen ${ }^{36}$. Nicht auszuschließen ist jedoch, dass die Oberkante der Lehmpackung ursprünglich ein älteres Geh- oder Nutzungsniveau bildete. Einen sicheren terminus post quem für diese Planierung gibt eine in SE 311 gefundene Münze des Caracalla (M 19), eine weitere Münze aus SE 313 war nicht zu bestimmen (M 18), eine hellenistische Münze (M 16) in dieser Schicht ist dagegen als Altstück anzusprechen ${ }^{37}$.

Im Bereich der unteren Treppenstufe des Raumeinganges befand sich unter SE 308 eine langgezogene, schmale und mit hartem, rötlichem opus caementitium verfüllte Schicht (SE 315), die als Baugrubenverfüllung der Treppenstufe anzusprechen ist.

Unter der beschriebenen Lehmpackung (SE 311/313) konnte eine weitere, zwischen 26,4 und 35,5 cm starke Planierung (SE 318/321/322/325) festgestellt werden, die aus sehr heterogenem Material ${ }^{38}$ bestand und sich über den gesamten Raum erstreckte. In ihr fanden sich zahlreiche große, Marmorimitation zeigende Wandmalereifragmente, welche mit den Beispielen aus der Wohneinheit 6 (Abb. 28, b) sehr gut zu vergleichen sind; des Weiteren stammt eine augusteische Prägung römischer Prokuratoren aus Judaea (M 23) aus SE 318: Es kann durchaus angenommen werden, dass während des Planierungsvorganges Material einer älteren, späthellenistischen oder frühkaiserzeitlichen Ausstattungsphase eingefüllt worden war ${ }^{39}$. Dafür spricht auch der hohe Anteil an teilweise sehr kleinteilig gebrochener späthellenistischer Keramik sowie zahlreicher linsen-

\footnotetext{
${ }^{36}$ Ein vergleichbarer Befund konnte in Raum 37 des Hanghauses 2 aufgedeckt werden (unpubl. Grabungen 1999).

37 s. dazu u. den Katalog der Münzen.

${ }^{38}$ Es handelte sich dabei wechselweise um grünlich gelblichen, festen Lehm, z. T. gräuliche, möglicherweise verwitterte und braune, sandige, mit vielen kleinen Steinen versetzte Erde.

${ }^{39}$ Vgl. auch die zeitliche Stellung der Wandmalereien, die in Sondage 4 West und Ost gefunden wurden.
} 


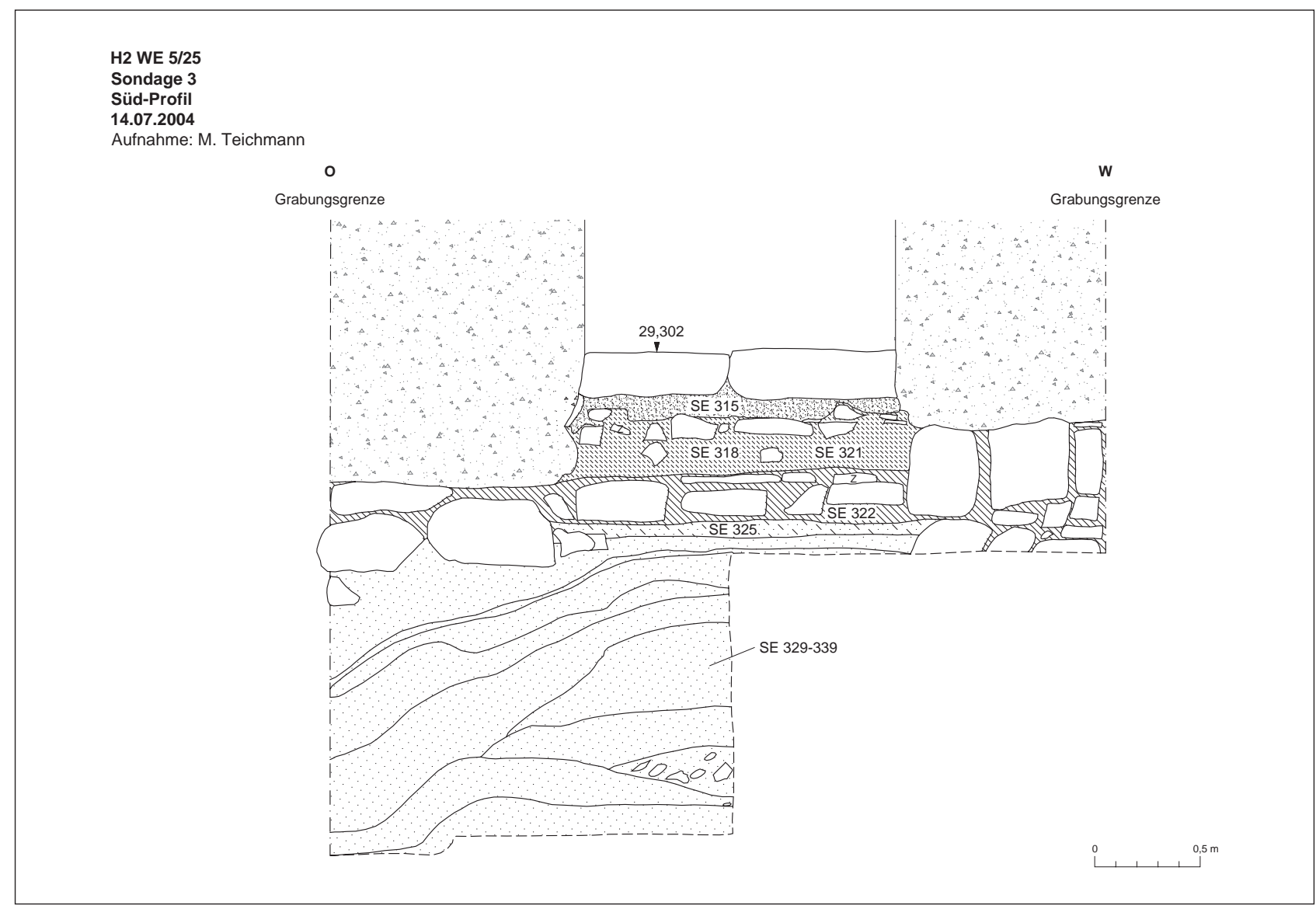

17 Südprofil und Ansicht der Südmauer (Bauphase I/IV) des Raumes 25

förmiger Webgewichte ${ }^{40}$ (Abb. 21, 10-12). Der Zeitpunkt dieser Arbeiten ist nicht auszumachen, lediglich die Münze gibt einen sicheren terminus post quem. Trotz des Fehlens eines repräsentativen Bodenbelages, der möglicherweise im Zuge späterer Umbaumaßnahmen entfernt wurde, darf demnach wohl davon ausgegangen werden, dass es sich um Planierungen handelt, die während der Bauphase I in den Raum eingebracht worden waren. Unter der Planierung SE 322/325 befand sich die Schicht SE 323, bestehend aus kompakter, brauner Erde und Bruchsteinen, die vor allem im mittleren westlichen Bereich größer waren.

Als Konstruktionselement der Basilika (Raum 8) der Wohneinheit 6 ist jene massive Packung (Abb. 16) anzusprechen, die parallel zur Ostmauer des Raumes aufgedeckt werden konnte (SE 319/320). Sie ist durchschnittlich 1,2 m stark, weist eine sauber horizontal abgestrichene Oberfläche auf und wurde im Westen gegen die anstehenden Erdschichten gesetzt, wodurch die unregelmäßige Kante in diesem Bereich zu erklären ist. Die Mörtelpackung besteht aus eingeschütteten Bruchsteinen, gebrannten, getrockneten Ziegeln und Ziegelbruch, die mit extrem harten Mörtel vergossen wurden, wobei zwischen dem eingefüllten Material z. T. größere Hohlräume bestehen blieben. Im Norden wurde die Packung von SE 330, einer rezenten, in den Raum hineinragenden Störung aus Beton, Ziegeln und Bruchsteinen, auf einer Fläche von $50 \times 30 \mathrm{~cm}_{\text {gestört }}{ }^{41}$. Bei SE 319/320 handelt es sich demnach um die Baugrubenverfüllung der Basilika, die in SE 321 einschneidet und damit jünger datiert ${ }^{42}$. Beim Abschluss der Grabungsarbeiten war in einer Tiefe von 1,36 m (im Süden) und 1,75 m (im Norden) die Unterkante der Packung noch nicht erreicht.

Dieser Befund macht deutlich, dass der Bau der Basilika die Bausubstanz von Raum 25 massiv beeinträchtigte, diente die massive Fundamentierung oder Füllung der Baugrube wohl nicht zuletzt einer Sicherung des

\footnotetext{
40 s. dazu E. Trinkl, Artefakte für die Textilbearbeitung, in: Lang-Auinger, Hanghaus 1, Funde 313-327.

${ }^{41}$ Es handelt sich dabei um Fundamentierungsarbeiten für die Stabilisierung des Tonnengewölbes im Zuge der Schutzdacherrichtung.

${ }^{42}$ Freundliche Mitteilung H. Thür.
} 


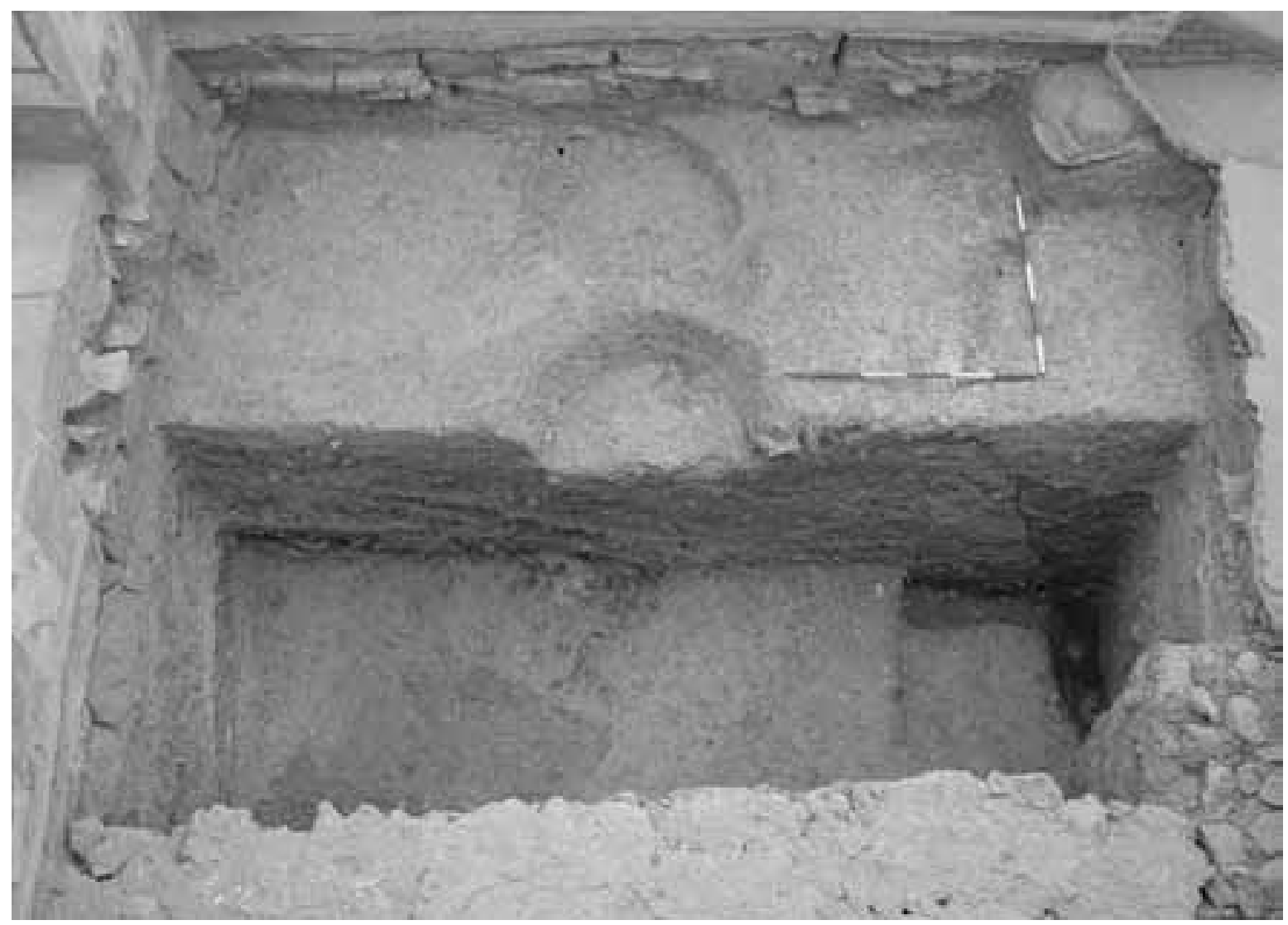

18 In die Aufschüttungen eingetiefte Gruben in Raum 25

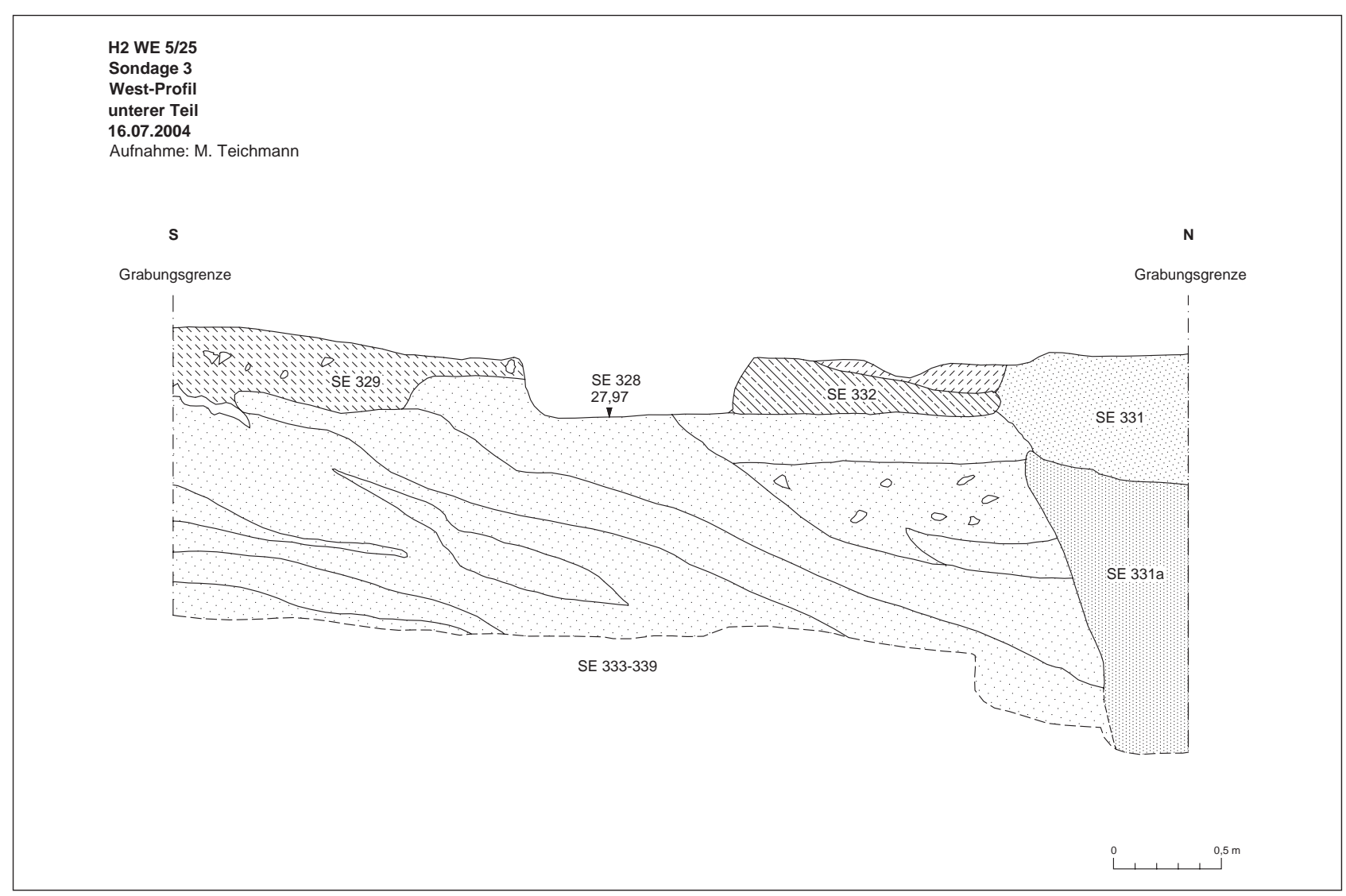

19 Westprofil der Sondage 3 
Tonnengewölbes. Die Errichtung der Basilika bedeutete für Raum 25 aber auch eine völlige Neuausstattung.

Entlang der Südmauer des Raumes konnte deren 22-30 cm tiefe Baugrube dokumentiert werden, die mit dunkelbraunem, lehmigem, hartem Material (SE 326) verfüllt war (Abb. 17). Sie entstand im Zuge von Bauphase I und korrespondiert mit dem Bruchsteinmauerwerk, von dem sich die untersten Lagen erhalten haben. Ein analoger Befund ließ sich auch entlang der - jedoch nicht fundamentierten - Westmauer beobachten.

Unter SE 325 befanden sich verfüllte Gruben, die in die Schicht SE 329 einschneiden, deren Funktion unklar bleiben muss. Bei SE 327 handelte es sich um eine etwa $90 \mathrm{~cm}$ breite, direkt an der

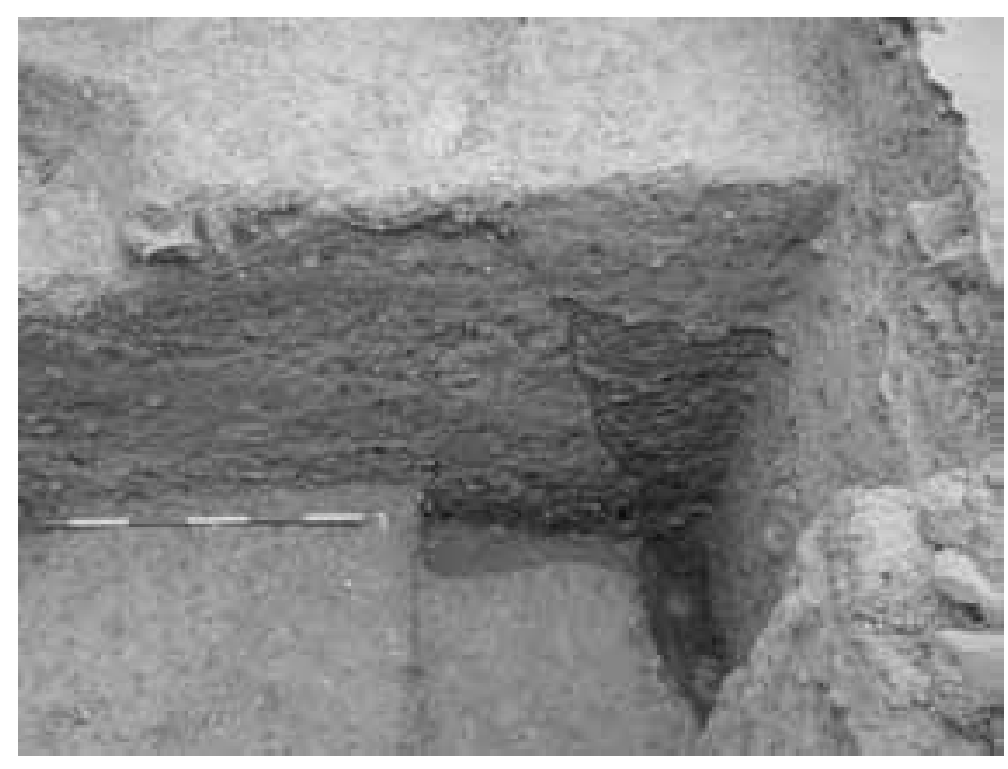

20 Baugruben der die Wohneinheit 5 im Norden begrenzenden Terrassenmauer Westmauer gelegene Grube, die sich ca. 1,40 m nördlich der Südmauer befand (Abb. 18). Die Grube war mit sandiger Erde, bräunlich grünlichen Lehmfragmenten, viel kleinteiligem Schiefersplitt und großen Bruchsteinen verfüllt. SE 328 war eine rundovale Grube, die leicht südlich der Raummitte lag; sie wies eine Verfüllung mit zahlreichen Bruchsteinen (mit einer durchschnittlichen Größe von etwa $15 \mathrm{~cm}$ ) und dunkelbrauner, lehmiger Erde auf.

Im weiteren Verlauf wurde die Grabungsfläche reduziert und die tiefer liegenden Planierschichten in einem lediglich $2 \mathrm{~m}$ breiten Streifen weiter verfolgt. Von besonderer Bedeutung sind dabei jene Einfüllungen (SE 332-339), die, wie das Westprofil deutlich zeigt (Abb. 19), von Süden her in den Raum eingeschüttet wurden, um eine Terrassierung des Areals zu erreichen. Die Schüttungen wurden etwa $3 \mathrm{~m}$ tief ausgehoben, ohne dass deren Unterkanten bei 25,9 m erreicht worden wären. Als Schuttmaterial fanden Schiefersplitt in unterschiedlichen Verwitterungsstufen, Lehm und eisenoxydhaltiges Erdmaterial Verwendung. Zudem handelt es sich um eine fundreiche Schicht, wobei insbesondere das keramische Material für die absolutchronologische Einordnung heranzuziehen ist. Erstaunlicherweise dominiert hellenistische Keramik des ausgehenden 3. und des beginnenden 2. Jahrhunderts, wogegen charakteristische Funde aus der zweiten Hälfte des 2. Jahrhunderts v. Chr. fehlen. Hinzuweisen ist auf Kratere im Westabhang-Nachfolgestil ${ }^{43}$ (Abb. 21, 4-5; 22, 4), Schälchen und Teller mit feinen, radial angeordneten Palmettenstempeln und Rouletting ${ }^{44}$ (Abb. 21, 6-8; 22, 5-7) sowie schwarz gefirnisste Skyphoi/Kantharoi (Abb. 21, 9). Von besonderer Bedeutung ist der Fund einer Münze (M 30), deren Prägezeit aufgrund des hier vorgelegten archäologischen Befundes in das frühe 2. Jahrhundert v. Chr. gerückt werden muss ${ }^{45}$.

Im Profil deutlich zu sehen ist auch, dass die Terrassenmauer mehrfach erneuert wurde (Abb. 20). Als Grund für diese Reparaturmaßnahmen, die erst nach einer Auswertung des keramischen Materials zeitlich eingeordnet werden können, dürfen möglicherweise von Erdbeben hervorgerufene Hangrutschungen im Areal des Hanghauses 2 genannt werden.

Zusammenfassend können für Raum 25 folgende Schlussfolgerungen gezogen werden: Das Areal wurde bereits gegen Ende des 3. Jahrhunderts v. Chr. oder im frühen 2. Jahrhundert v. Chr. planiert. In diesen Zeitraum datiert demnach wohl auch die im Norden die Terrasse begrenzende Mauer. Es handelt sich um die ältesten Befunde, die auf dem Bauplatz des Hanghauses 2 dokumentiert werden konnten. Einer frühkaiser-

\footnotetext{
${ }^{43}$ Zum ephesischen Bestand s. vor allem V. Mitspoulos-Leon, Die Basilika am Staatsmarkt in Ephesos. Kleinfunde. 1. Teil: Keramik hellenistischer und römischer Zeit, FiE IX 2, 2 (1991) 39 f. Zur Datierung s. S. Rotroff, Hellenistic Pottery. Athenian and imported wheelmade table ware and related material, Agora XXIX (1997) 138.

44 Zahlreiche Exemplare finden sich am Staatsmarkt in Ephesos: Mitsopoulos-Leon (Anm. 43) A 14 (Echinusschälchen). A 81. A 82. A 86 (gestempelte Bodenfragmente).

45 s. dazu u. zu den Fundmünzen.
} 
1
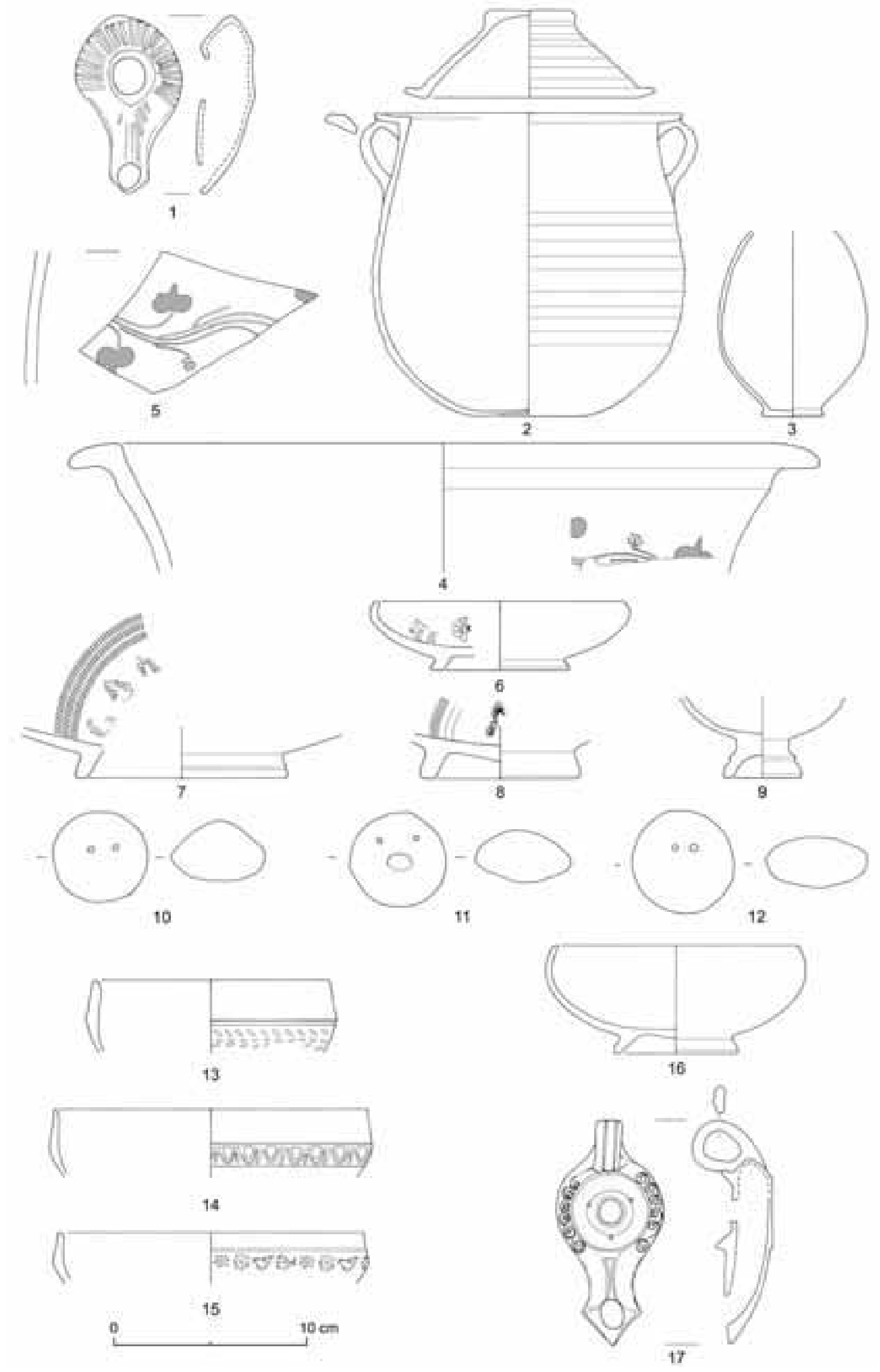

21 Ausgewählte Keramikfunde aus den Sondagen 1-4 

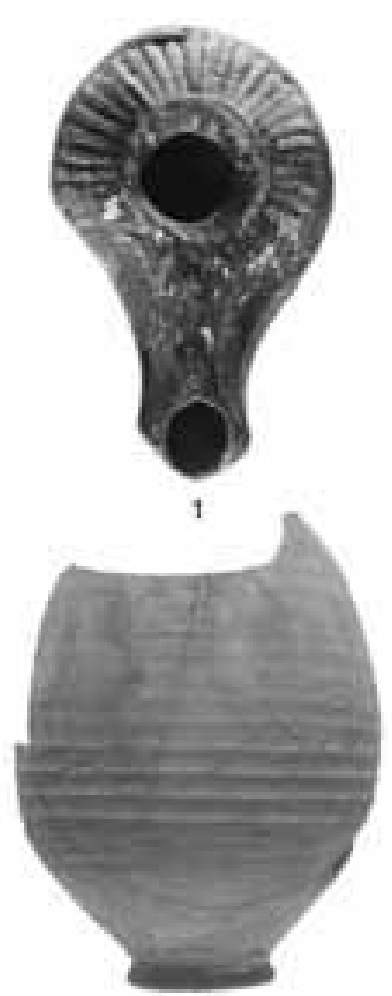

3
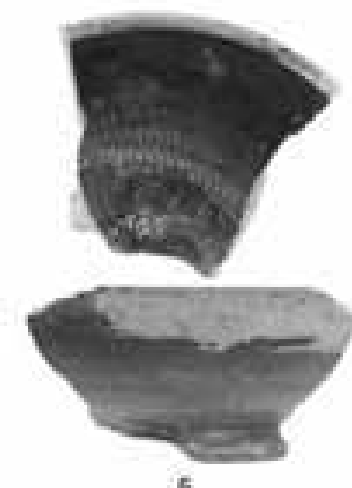

5
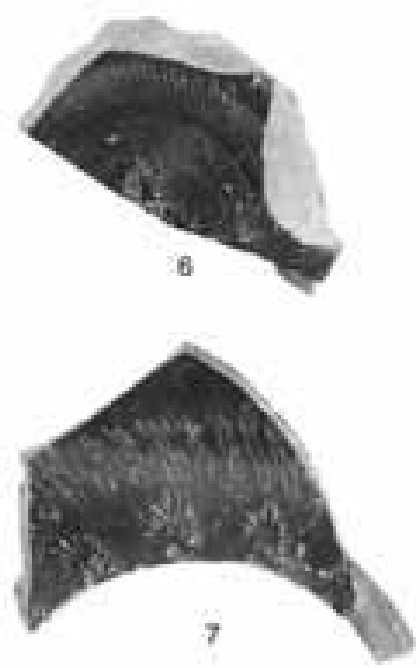

7
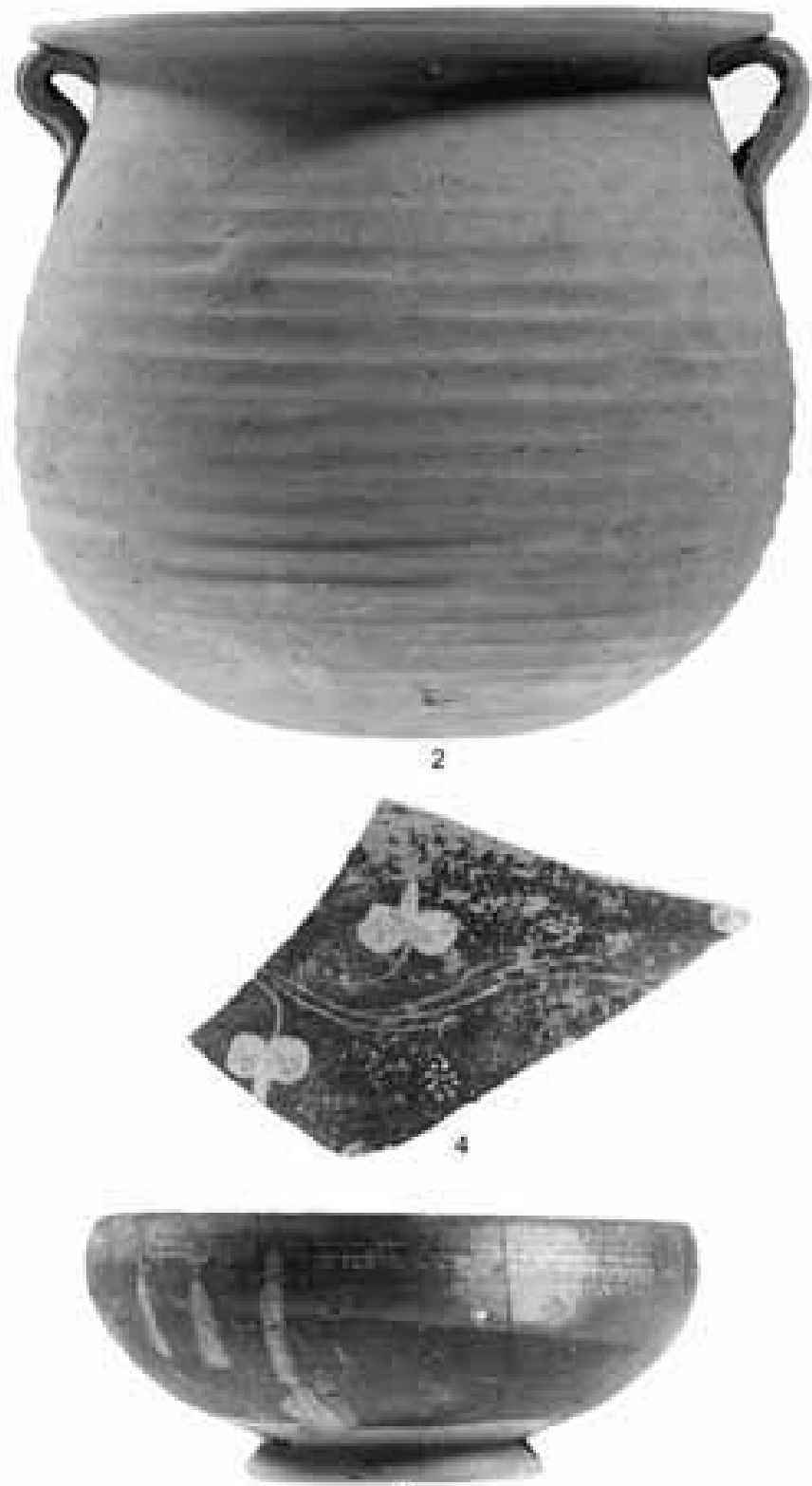

8
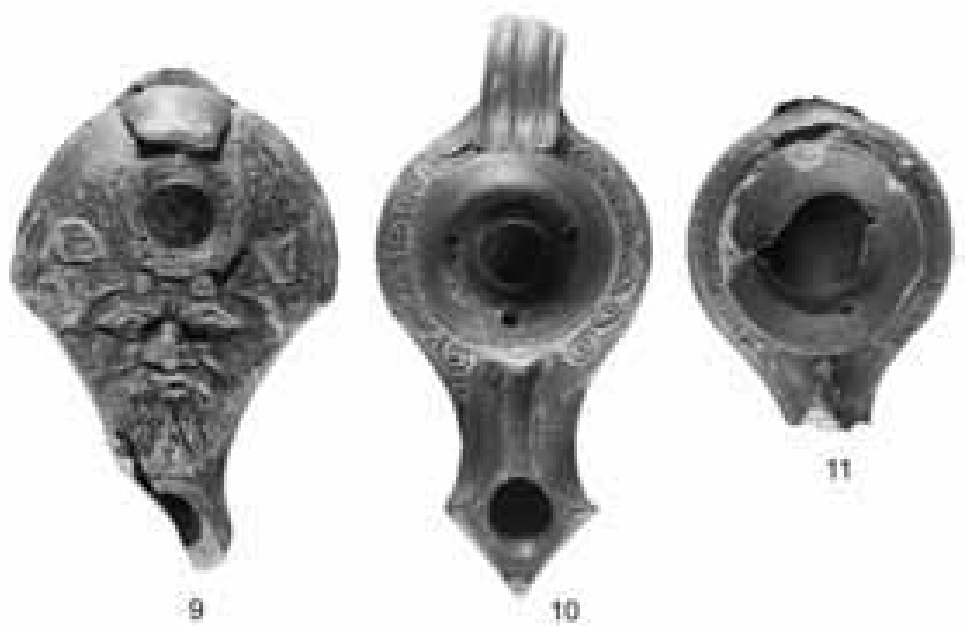

22 Ausgewählte Keramikfunde aus den Sondagen 1-4 


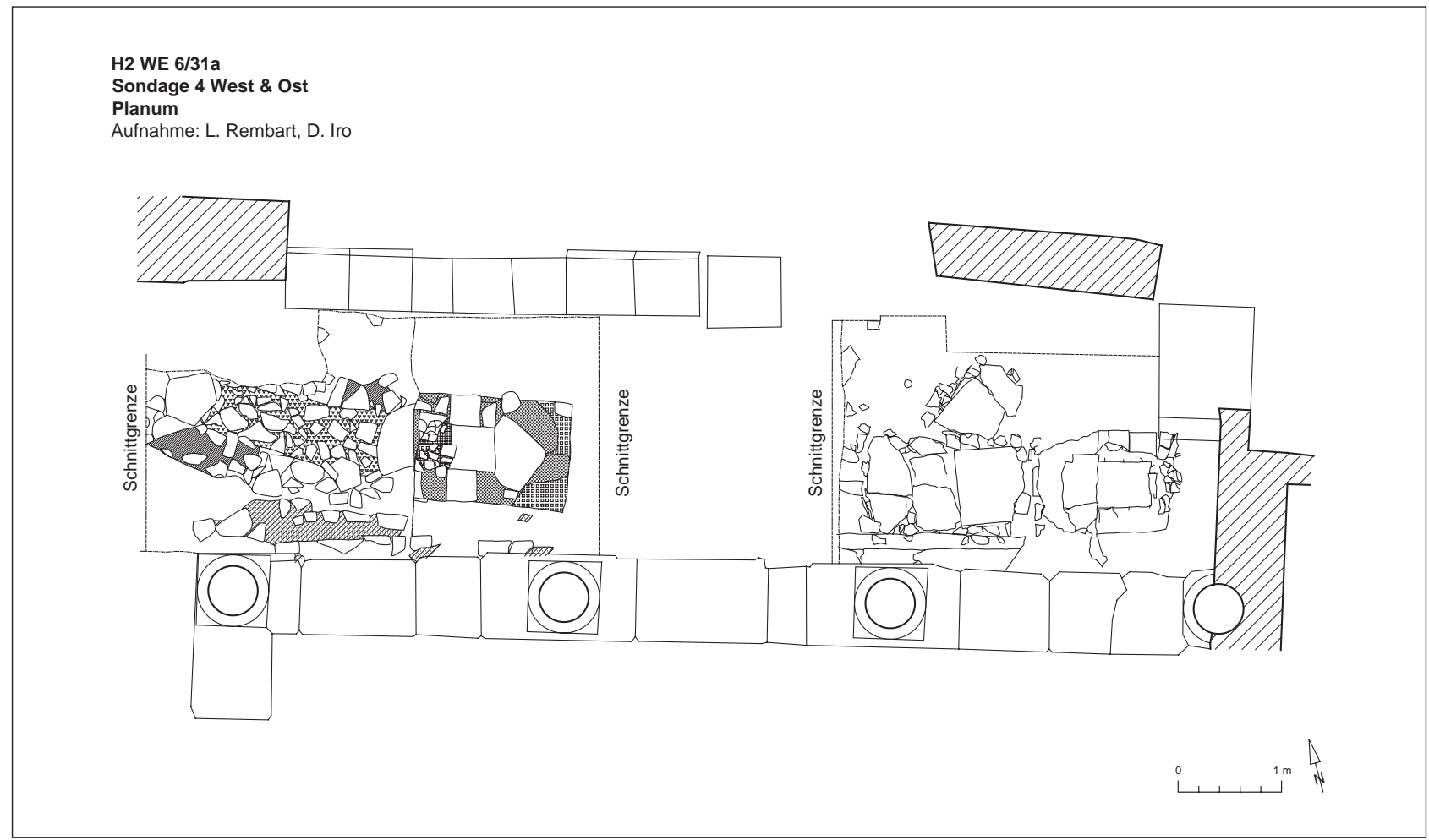

23 Kanalsystem im Nordumgang des Peristylhofs 31a der Wohneinheit 6 (Sondage 4)

zeitlichen Bauphase dagegen gehört jene Planierung an, die zahlreiche Wandmalereifragmente enthielt; deren Existenz lässt jedoch auf einen älteren Wohnbau an dieser Stelle schließen. Anlässlich des Einbaus der Basilika in Bauphase III wurde in den Raum massiv eingegriffen und eine stabile Konstruktion für deren gewölbte Tonne geschaffen. Die darüber liegenden Planierungen datieren in Bauphase III und IV und geben wichtige Anhaltspunkte für die zeitliche Einordnung der Ausstattungselemente.

\section{Sondage 4/Wohneinheit 6 - Peristylhof 31a}

Im Nordumgang des Peristylhofs der Wohneinheit 6 wurden zwei weitere Schnitte angelegt. Diese Untersuchungen setzten eine Abnahme des z. T. stark zerstörten Marmorplattenbodens voraus. Begrenzt wurden die Flächen im Süden von dem Stylobat des Hofs sowie im Norden von den hier liegenden Räumen, ausgespart blieben darüber hinaus jene Bereiche, die bereits anlässlich von Anastylosearbeiten und Fundamentuntersuchungen im Jahr 1988 ergraben und mit Beton vergossen worden waren.

\section{Sondage 4/04 West}

Der hier flächig verlegte Marmorplattenboden wies eine homogene dreilagige Unterkonstruktion, bestehend aus einem Ziegelsplittestrich (SE 402) und einer in weißlich grauen Kalkmörtel (SE 405) gebetteten Ziegelbruchrollierung (SE 403) auf. Unter einer 15 cm dicken Erdplanierung (SE 406) zeigte sich ein im Planum deutlich sichtbares, West-Ost verlaufendes und durchschnittlich 1,15 m breites Stratum (SE 409), das in weiterer Folge als Grubenverfüllung interpretiert werden konnte. Der Grund für den Aushub der Grube liegt in Reparaturarbeiten eines in einer Tiefe von $55 \mathrm{~cm}$ unter dem Stylobat angetroffenen West-Ost laufenden Kanals (Abb. 23), bei denen wohl die Abdeckplatten ersetzt wurden ${ }^{46}$. Die Maßnahmen datieren nach Ausweis eines

${ }^{46}$ Mit diesem Vorgang ist die unregelmäßige Bruchsteinauflage der Kanalwangen zu erklären, während am Mauerwerk selbst scheinbar keine Ausbesserungsarbeiten notwendig waren. 